\title{
Upper bound of the proton lifetime in product-group unification
}

\author{
Masahiro Ibe and T. Watari \\ Department of Physics, University of Tokyo, Tokyo 113-0033, Japan
}

(Received 18 March 2003; published 23 June 2003)

\begin{abstract}
Models of supersymmetric grand unified theories based on the $\mathrm{SU}(5)_{\mathrm{GUT}} \times \mathrm{U}(N)_{H}$ gauge group $(N=2,3)$ have a symmetry that guarantees light Higgs doublets and the absence of dimension-5 proton-decay operators. We analyze the proton decay induced by the gauge-boson exchange in these models. Upper bounds on the proton lifetime are obtained: $\tau\left(p \rightarrow \pi^{0} e^{+}\right) \leqq 6.0 \times 10^{33} \mathrm{yr}$ in the $\mathrm{SU}(5)_{\mathrm{GUT}} \times \mathrm{U}(2)_{H}$ model and $\tau\left(p \rightarrow \pi^{0} e^{+}\right)$ $\leqslant 5.3 \times 10^{35} \mathrm{yr}$ in the $\mathrm{SU}(5)_{\mathrm{GUT}} \times \mathrm{U}(3)_{H}$ model. Various uncertainties in the predictions are also discussed.
\end{abstract}

DOI: 10.1103/PhysRevD.67.114021

PACS number(s): 13.30.-a, 12.10.Dm, 12.60.Jv

\section{INTRODUCTION}

The supersymmetric (SUSY) grand unified theories (GUTs) are among some of the most promising candidates for physics beyond the standard model (SM); this is because of their theoretical beauty and also because the gaugecoupling unification is supported by precision experiments. The GUTs generically predict proton decay through a gaugeboson exchange. ${ }^{1}$ The lifetime of the proton, however, varies very much from model to model; it is proportional to the fourth power of the gauge-boson mass. Thus, the protondecay experiments are able not only to provide strong support for the GUTs but also to select some models out of several candidates.

For more than 20 years, many attempts have been made at constructing models of SUSY GUTs. There are two strong hints to finding realistic models. First, the two Higgs doublets are light, whereas their mass term is not forbidden by any of the gauge symmetries of the minimal supersymmetric standard model (MSSM). Second, the rate of proton decay through dimension-5 operators [2] is smaller than naturally expected [3]. ${ }^{2}$ Thus, the two phenomena require two small parameters; it would then, be conventional wisdom to consider that there might be symmetries behind the small parameters. Moreover, only one symmetry is sufficient to explain the two phenomena for the following reason. If a symmetry forbids the mass term of the two Higgs doublets,

$$
W \boxplus H_{d} H_{u}
$$

then the dimension-5 proton-decay operators are also forbidden by the same symmetry,

$$
W \boxplus Q Q Q L+\bar{U} \bar{E} \bar{U} \bar{D}
$$

and vice versa. Here, it is implicitly assumed that the quarks and leptons have the same charge under the symmetry when

\footnotetext{
${ }^{1}$ There is a class of models of SUSY GUTs where the gaugeboson exchange does not induce proton decay [1].

${ }^{2}$ The experimental lower bound of the proton lifetime through these operators is $\tau\left(p \rightarrow K^{+} \bar{\nu}\right) \geq 6.7 \times 10^{32} \mathrm{yr}(90 \%$ C.L.) [4].
}

they are in the same SU(5) multiplet, and that the Yukawa couplings of quarks and leptons are allowed by the symmetry.

There are three classes of models that have such a symmetry. ${ }^{3}$ One uses the $\mathrm{SU}(5)_{1} \times \mathrm{SU}(5)_{2}$ gauge group [10-12], where there is an unbroken $\mathbf{Z}_{N}$ symmetry $[7,12]$. The second class consists of models based on the $\mathrm{SU}(5)_{\mathrm{GUT}} \times \mathrm{U}(N)_{H}(N=2,3)$ gauge group $[16,17,13]$, where there is an unbroken discrete $R$ symmetry [13]. The last class of models can be constructed so that the unified gauge group is a simple group [18]. The symmetry discussed in the previous paragraph, however, cannot remain unbroken; it is broken in such a way that the dimension-5 operators are not completely forbidden. The proton decay through gaugeboson exchange is discussed in $[19,20]$ for these models.

In this article, we analyze the proton decay for the second class of models. The dimension-5 operators are completely forbidden in these models, and the proton decay is induced by gauge-boson exchange. Our analysis is based on the models in [13], which use the $\mathrm{SU}(5)_{\mathrm{GUT}} \times \mathrm{U}(N)_{H}$ gauge group $(N=2,3)$. Reference [21] obtained an estimate of the proton lifetime in the $\mathrm{SU}(5)_{\mathrm{GUT}} \times \mathrm{U}(3)_{H}$ model, adopting a number of Ansätze to make the analysis simple. The estimate was ${ }^{4}$ $\tau\left(p \rightarrow \pi^{0} e^{+}\right) \simeq(0.7-3) \times 10^{34} \mathrm{yr}$, and hence there is an intriguing possibility that the proton decay can be observed in the next generation of water Cerenkov detectors. This article presents a full analysis: both the $\mathrm{SU}(5)_{\mathrm{GUT}} \times \mathrm{U}(2)_{H}$ model and the $\mathrm{SU}(5)_{\mathrm{GUT}} \times \mathrm{U}(3)_{H}$ one are analyzed without any Ansätze. Three parameters of the models are fixed by three gauge coupling constants of the MSSM, and the remaining parameters are left undetermined. The range of these parameters is restricted when the models are required to be in the calculable regime. As a result, the range of the gauge-boson mass is restricted. Thus, we obtain the range $e^{5}$ of the lifetime rather than an estimate of it.

\footnotetext{
${ }^{3}$ There are other types of models that are not constructed as field theoretical models in four-dimensional space-time [5,6], [7] and $[8,9]$. Reference [7] is a string-theoretical realization of [10-12] and Refs. [8,9] are higher-dimensional extensions of [13]. The proton decay is analyzed in $[6,14]$ for the model [5] and in [15] for the model [7].

${ }^{4}$ The following numerical value is based on a reanalysis in [22]

${ }^{5}$ This procedure is the one adopted in [23], where the minimal SU(5) SUSY GUT model was analyzed.
} 
The organization of this article is as follows. First, we briefly review both the $\mathrm{SU}(5)_{\mathrm{GUT}} \times \mathrm{U}(N)_{H}$ models $(N$ $=2,3$ ) in Sec. II. The range of the GUT gauge-boson mass is determined both for the $\mathrm{SU}(5)_{\mathrm{GUT}} \times \mathrm{U}(2)_{H}$ model and for the $\mathrm{SU}(5)_{\mathrm{GUT}} \times \mathrm{U}(3)_{H}$ model in Secs. III and IV, respectively. In particular, it is shown that the range of the mass is bounded from above, which leads to an upper bound of the lifetime of the proton in each model. The upper bounds ${ }^{6}$ are, in general, predictions that can be confirmed by experiments. Since the SUSY-particle spectrum affects the MSSM gaugecoupling constants through threshold corrections, the upper bound of the lifetime depends on the spectrum. Therefore, the upper bound is shown as a function of SUSY-breaking parameters in Sec. V for both models. Various uncertainties in our predictions are also discussed. Section VI gives a brief summary of the results obtained in this article and compares the results with predictions of other models. We find that $\tau\left(p \rightarrow \pi^{0} e^{+}\right) \leq 6.0 \times 10^{33}$ yr in the $\mathrm{SU}(5)_{\mathrm{GUT}} \times \mathrm{U}(2)_{H}$ model and $\tau\left(p \rightarrow \pi^{0} e^{+}\right) \leq 5.3 \times 10^{35} \mathrm{yr}$ in the $\mathrm{SU}(5)_{\mathrm{GUT}} \times \mathrm{U}(3)_{H}$ model; here, we exploit the uncertainties in the value of the QCD coupling constant (by $\pm 2 \sigma$ ) and in the threshold corrections from the SUSY particles; other uncertainties, which cannot be estimated, are not included in these figures.

\section{BRIEF REVIEW OF MODELS}

\section{A. $\mathrm{SU}(5)_{\mathrm{GUT}} \times \mathrm{U}(2)_{H}$ model}

Let us first explain a model based on the product gauge group $\mathrm{SU}(5)_{\mathrm{GUT}} \times \mathrm{U}(2)_{H}$. Quarks and leptons are singlets of the $\mathrm{U}(2)_{H}$ gauge group and form three families of $\mathbf{5}^{*}$ +10 of the $\mathrm{SU}(5)_{\mathrm{GUT}}$. Fields introduced to break the $\mathrm{SU}(5)_{\mathrm{GUT}}$ symmetry are given as follows: $X_{\beta}^{\alpha}(\alpha, \beta=1,2)$, which transforms as $(\mathbf{1}, \mathbf{a d j}=\mathbf{3}+\mathbf{1})$ under the $\mathrm{SU}(5)_{\mathrm{GUT}}$ $\times \mathrm{U}(2)_{H}$ gauge group, and $Q_{i}^{\alpha}(i=1, \ldots, 5)+Q_{6}^{\alpha}$ and $\bar{Q}_{\alpha}^{i}$ $(i=1, \ldots, 5)+\bar{Q}_{\alpha}^{6}$, which transform as $\left(\mathbf{5}^{*}+\mathbf{1}, \mathbf{2}\right)$ and $(\mathbf{5}$ $\left.+\mathbf{1}, \mathbf{2}^{*}\right)$. The ordinary Higgs fields $H^{i}(\mathbf{5})$ and $\bar{H}_{i}\left(\mathbf{5}^{*}\right)$ are not introduced; the fields $Q_{6}^{\alpha}$ and $\bar{Q}_{\alpha}^{6}$ are eventually identified with the two Higgs doublets. The $\mathrm{SU}(5)_{\mathrm{GUT}}$ index is denoted by $i$ and the $\mathrm{U}(2)_{H}$ index by $\alpha$ or $\beta$. The chiral superfield $X_{\beta}^{\alpha}$ is also written as $X^{c}\left(t_{c}\right)_{\beta}^{\alpha}(c=0,1,2,3)$, where $t_{a}(a$ $=1,2,3)$ are Pauli matrices of the $\mathrm{SU}(2)_{H}$ gauge group ${ }^{7}$ and $t_{0} \equiv \mathbf{1}_{2 \times 2} / 2$, where $\mathrm{U}(2)_{H} \simeq \mathrm{SU}(2)_{H} \times \mathrm{U}(1)_{H}$. The $(\bmod 4)$ $R$ charge assignment of all the fields in this model is summarized in Table I. This symmetry forbids both the enormous mass term ${ }^{8} W=Q_{6}^{\alpha} \bar{Q}_{\alpha}^{6}$ and the dangerous dimension-5 proton-decay operators $W=\mathbf{1 0} \cdot \mathbf{1 0} \cdot \mathbf{1 0} \cdot \mathbf{5}^{*}$.

The most generic superpotential under the $R$ symmetry is given by

${ }^{6}$ Only a lower bound is obtained, e.g., in the minimal SU(5) model [23].

${ }^{7}$ The normalization condition $\operatorname{tr}\left(t_{a} t_{b}\right)=\delta_{a b} / 2$ is understood. Note that the normalization of the following $t_{0}$ is determined in such a way that it also satisfies $\operatorname{tr}\left(t_{0} t_{0}\right)=1 / 2$.

${ }^{8}$ The mass term of the order of the weak scale for the Higgs doublets can be obtained through the Giudice-Masiero mechanism [24].
TABLE I. (Mod 4) $R$ charges of the fields in the $\mathrm{SU}(5)_{\mathrm{GUT}}$ $\times \mathrm{U}(2)_{H}$ model.

$$
\begin{aligned}
& \begin{array}{lcrrcc}
\hline \hline \text { Fields } & \mathbf{1 0}^{i j} & \mathbf{5}_{i}^{*} & X & Q_{i}, \bar{Q}^{i} & Q_{6}, \bar{Q}^{6} \\
R \text { charges } & 1 & 1 & 2 & 0 & 0 \\
\hline \hline
\end{array} \\
& W=\sqrt{2} \lambda_{2 H} \bar{Q}^{i}{ }_{\alpha} X^{a}\left(t_{a}\right)^{\alpha}{ }_{\beta} Q^{\beta}{ }_{i}+\sqrt{2} \lambda_{2 \mathrm{H}} \bar{Q}^{6}{ }_{\alpha} X^{a}\left(t_{a}\right)^{\alpha}{ }_{\beta} Q^{\beta}{ }_{6} \\
& +\sqrt{2} \lambda_{1 H} \bar{Q}^{i}{ }_{\alpha} X^{0}\left(t_{0}\right)^{\alpha}{ }_{\beta} Q^{\beta}{ }_{i}+\sqrt{2} \lambda_{1 \mathrm{H}}^{\prime} \bar{Q}^{6}{ }_{\alpha} X^{0}\left(t_{0}\right)^{\alpha}{ }_{\beta} Q^{\beta}{ }_{6} \\
& -\sqrt{2} \lambda_{1 H} v^{2} X_{\alpha}^{\alpha}+c_{\mathbf{1 0}} \mathbf{1 0}^{i_{1} i_{2}} \mathbf{1 0}^{i_{3} i_{4}}(\bar{Q} Q)_{6}^{i_{5}} \\
& +c_{\mathbf{5} *}(\bar{Q} Q)^{6}{ }_{i} \cdot \mathbf{1 0}^{i j} \cdot \mathbf{5}_{j}^{*}+\cdots,
\end{aligned}
$$

where the parameter $v$ is taken to be of the order of the GUT scale; $\lambda_{2 H}, \lambda_{2 H}^{\prime}, \lambda_{1 H}$, and $\lambda_{1 H}^{\prime}$ are dimensionless coupling constants; $c_{\mathbf{1 0}}$ and $c_{\mathbf{5}} *$ have dimensions of (mass) ${ }^{-1}$. The ellipses stand for neutrino-mass terms and other nonrenormalizable terms. The fields $Q_{i}^{\alpha}$ and $\bar{Q}_{\alpha}^{i}$ in the bifundamental representation acquire vacuum expectation values (VEVs) $\left\langle Q_{i}^{\alpha}\right\rangle=v \delta_{i}^{\alpha}$ and $\left\langle\bar{Q}_{\alpha}^{i}\right\rangle=v \delta_{\alpha}^{i}$, because of the first three lines in Eq. (3). Thus, the gauge group $\mathrm{SU}(5)_{\mathrm{GUT}} \times \mathrm{U}(2)_{H}$ is broken down to that of the SM. The first and third terms in Eq. (3) provide mass terms for the unwanted particles. $Q_{6}^{\alpha}$ and $\bar{Q}_{\alpha}^{6}$ are identified with the Higgs doublets in this model, and no Higgs triplets appear in the spectrum. As a result, no particle other than the MSSM fields, not even a gauge singlet of the MSSM, remains in the low-energy spectrum. This fact guarantees not only that the gauge coupling unification of the MSSM is maintained, but also that the vacuum is isolated. The $R$ symmetry is not broken at the GUT scale, and the $\mu$ term, dimension-4 and dimension-5 proton-decay operators, are forbidden by this unbroken symmetry.

The fine structure constants of the MSSM are given at the tree level by

$$
\begin{aligned}
& \frac{1}{\alpha_{3}} \equiv \frac{1}{\alpha_{C}}=\frac{1}{\alpha_{\mathrm{GUT}}}, \\
& \frac{1}{\alpha_{2}} \equiv \frac{1}{\alpha_{L}}=\frac{1}{\alpha_{\mathrm{GUT}}}+\frac{1}{\alpha_{2 H}},
\end{aligned}
$$

and

$$
\frac{1}{\alpha_{1}} \equiv \frac{3 / 5}{\alpha_{Y}}=\frac{1}{\alpha_{\mathrm{GUT}}}+\frac{3 / 5}{\alpha_{1 H}},
$$

where $\alpha_{\mathrm{GUT}}, \alpha_{2 H}$, and $\alpha_{1 H}$ are the fine structure constants of $\mathrm{SU}(5)_{\mathrm{GUT}}, \mathrm{SU}(2)_{H}$, and $\mathrm{U}(1)_{H}$, respectively. Thus, the approximate unification of $\alpha_{3}, \alpha_{2}$, and $\alpha_{1}$ is maintained when $\alpha_{2 H}$ and $\alpha_{1 H}$ are sufficiently large.

Although it is true that the gauge-coupling unification is no longer a generic prediction of this model, nevertheless it need not be a mere coincidence. Here, the gauge-coupling unification is a consequence of the fact that $\alpha_{2 H}$ and $\alpha_{1 H}$ are relatively strongly coupled when compared with $\alpha_{\mathrm{GUT}}$. 
TABLE II. (Mod 4) $R$ charges of the fields in the $\mathrm{SU}(5)_{\mathrm{GUT}}$ $\times \mathrm{U}(3)_{H}$ model.

\begin{tabular}{lccccccccc}
\hline \hline Fields & $\mathbf{1 0}^{i j}$ & $\mathbf{5}_{i}^{*}$ & $H(\mathbf{5})^{i}$ & $\bar{H}\left(\mathbf{5}^{*}\right)_{i}$ & $X^{\alpha}{ }_{\beta}$ & $Q^{\alpha}{ }_{i}$ & $\bar{Q}^{i}{ }_{\alpha}$ & $Q_{6}^{\alpha}$ & $\bar{Q}^{6}{ }_{\alpha}$ \\
$R$ charges & 1 & 1 & 0 & 0 & 2 & 0 & 0 & 2 & -2 \\
\hline \hline
\end{tabular}

\section{B. $\mathrm{SU}(5)_{\mathrm{GUT}} \times \mathrm{U}(3)_{H}$ model}

The other model is based on an $\mathrm{SU}(5)_{\mathrm{GUT}} \times \mathrm{U}(3)_{H}$ gauge group, where $\mathrm{U}(3)_{H} \simeq \mathrm{SU}(3)_{H} \times \mathrm{U}(1)_{H}$. Under the $\mathrm{SU}(5)_{\mathrm{GUT}} \times \mathrm{U}(3)_{H}$ gauge group, the particle content of this model is $\bar{Q}_{\alpha}^{k}\left(\mathbf{5}+\mathbf{1}, \mathbf{3}^{*}\right), Q_{k}^{\alpha}\left(\mathbf{5}^{*}+\mathbf{1}, \mathbf{3}\right)$, and $X_{\beta}^{\alpha}(\mathbf{1}, \mathbf{a d j}=\mathbf{3}$ $\left.\otimes \mathbf{3}^{*}\right)$, with $k=1, \ldots, 5,6 ; \alpha, \beta=1,2,3$. In addition, there are the three ordinary families of quarks and leptons $\left(5^{*}\right.$ $+\mathbf{1 0}, \mathbf{1})$ and Higgs multiplets $H^{i}+\bar{H}_{i}\left(\mathbf{5}+\mathbf{5}^{*}, \mathbf{1}\right)$. The $R$ symmetry forbids both (1) and (2). The $R$ charges of the fields are summarized in Table II.

The most generic superpotential under the $R$ symmetry is given by [13]

$$
\begin{aligned}
W= & \sqrt{2} \lambda_{3 H} \bar{Q}^{i}{ }_{\alpha} X^{a}\left(t_{a}\right)^{\alpha}{ }_{\beta} Q^{\beta}{ }_{i}+\sqrt{2} \lambda_{3 H}^{\prime} \bar{Q}^{6}{ }_{\alpha} X^{a}\left(t_{a}\right)_{\beta}^{\alpha} Q^{\beta}{ }_{6} \\
& +\sqrt{2} \lambda_{1 H} \bar{Q}^{i}{ }_{\alpha} X^{0}\left(t_{0}\right)^{\alpha}{ }_{\beta} Q^{\beta}{ }_{i}+\sqrt{2} \lambda_{1 \mathrm{H}}^{\prime} \bar{Q}^{6}{ }_{\alpha} X^{0}\left(t_{0}\right)_{\beta}^{\alpha} Q^{\beta}{ }_{6} \\
& -\sqrt{2} \lambda_{1 H} v^{2} X^{\alpha}{ }_{\alpha}+h^{\prime} \bar{H}_{i} \bar{Q}^{i}{ }_{\alpha} Q^{\alpha}{ }_{6}+h \bar{Q}^{6}{ }_{\alpha} Q^{\alpha}{ }_{i} H^{i} \\
& +y_{\mathbf{1 0}} \mathbf{1 0} \cdot \mathbf{1 0} \cdot H+y_{\mathbf{5}^{*}} \mathbf{5}^{*} \cdot \mathbf{1 0} \cdot \bar{H}+\cdots,
\end{aligned}
$$

where $t_{a}(a=1,2, \ldots, 8)$ are Gell-Mann matrices, $t_{0}$ $\equiv \mathbf{1}_{3 \times 3} / \sqrt{6}, y_{10}$ and $y_{5} *$ are Yukawa coupling constants of the quarks and leptons, and $\lambda_{3 H}, \lambda_{3 H}^{\prime}, \lambda_{1 H}, \lambda_{1 H}^{\prime}, h^{\prime}$, and $h$ are dimensionless coupling constants. The first three lines of Eq. (7) lead to the desirable VEV of the form $\left\langle Q_{i}^{\alpha}\right\rangle=v \delta_{i}^{\alpha}$ and $\left\langle\bar{Q}_{\alpha}^{i}\right\rangle=v \delta_{\alpha}^{i}$. Thus, the $\mathrm{SU}(5)_{\mathrm{GUT}} \times \mathrm{U}(3)_{H}$ gauge group is broken down to that of the SM. The mass terms of the colored Higgs multiplets arise from the fourth line in Eq. (7) in the GUT-symmetry-breaking vacuum. No unwanted particle remains massless.

The fine structure constants of the $\mathrm{SU}(3)_{H} \times \mathrm{U}(1)_{H}$ groups must be larger than that of the $\mathrm{SU}(5)_{\mathrm{GUT}}$. This is because the gauge-coupling constants of the MSSM are given by

$$
\begin{aligned}
& \frac{1}{\alpha_{3}} \equiv \frac{1}{\alpha_{C}}=\frac{1}{\alpha_{\mathrm{GUT}}}+\frac{1}{\alpha_{3 H}}, \\
& \frac{1}{\alpha_{2}} \equiv \frac{1}{\alpha_{L}}=\frac{1}{\alpha_{\mathrm{GUT}}},
\end{aligned}
$$

and

$$
\frac{1}{\alpha_{1}} \equiv \frac{3 / 5}{\alpha_{Y}}=\frac{1}{\alpha_{\mathrm{GUT}}}+\frac{2 / 5}{\alpha_{1 H}},
$$

where $\alpha_{3 H}$ and $\alpha_{1 H}$ are the fine structure constants of $\mathrm{SU}(3)_{H}$ and $\mathrm{U}(1)_{H}$, respectively. Thus, the approximate unification of $\alpha_{3}, \alpha_{2}$, and $\alpha_{1}$ is maintained when $\alpha_{3 H}$ and $\alpha_{1 H}$ are sufficiently large.

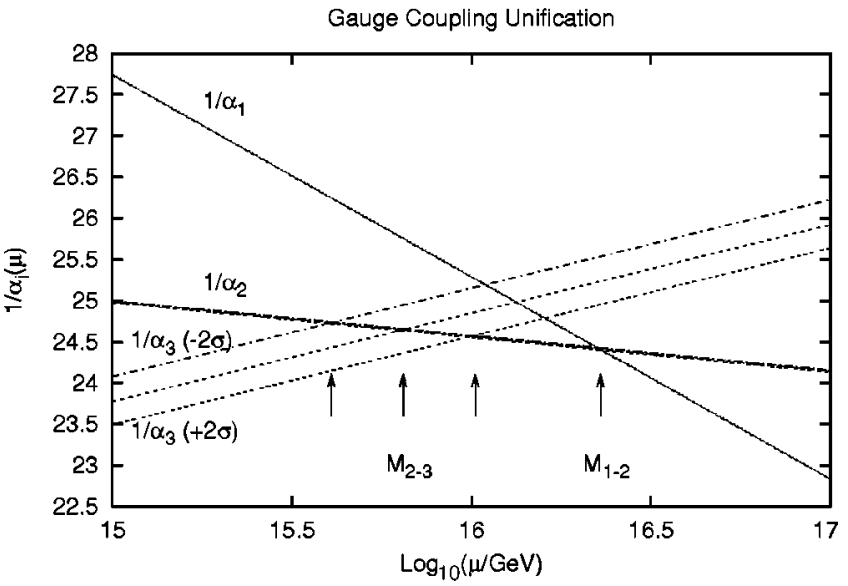

FIG. 1. Close-up view of the unification of the three gaugecoupling constants of the MSSM. The fine structure constants in the $\overline{\mathrm{DR}}$ scheme of the $\mathrm{U}(1)_{Y}, \mathrm{SU}(2)_{L}$, and $\mathrm{SU}(3)_{C}$ gauge interactions are denoted by $\alpha_{1,2,3}$, respectively. The three lines of $\alpha_{3}$ correspond to three different experimental inputs; the QCD coupling constants $\alpha_{s}^{\overline{\mathrm{MS}},(5)}\left(M_{Z}\right)=0.1132(-2 \sigma), 0.1172$ (central value), and 0.1212 $(+2 \sigma)$ are used [29]. The two-loop renormalization-group effects of the MSSM and the SUSY threshold corrections are taken into account. The latter corrections are those from the SUSY-particle spectrum determined by the MSUGRA boundary condition with $\tan \beta=10, A_{0}=0 \mathrm{GeV},\left(m_{0}, m_{1 / 2}\right)=(400 \mathrm{GeV}, 300 \mathrm{GeV})$, and $\mu$ $>0$ (see the caption for Fig. 4 below for the convention on the sign of $\mu)$.

\section{Rough estimate of matching scale}

Figure 1 shows the renormalization-group evolution of the three gauge-coupling constants of the MSSM. The treelevel matching equations (4) - (6) and (8) - (10) suggest that the matching scale is below the energy scale $M_{2-3}$ in Fig. 1 in the $\mathrm{SU}(5)_{\mathrm{GUT}} \times \mathrm{U}(2)_{H}$ model, and is between the two energy scales $M_{2-3}$ and $M_{1-2}$ in the $\mathrm{SU}(5)_{\mathrm{GUT}} \times \mathrm{U}(3)_{H}$ model. Here, $M_{2-3}$ is the energy scale where the coupling constants of $\mathrm{SU}(2)_{L}$ and $\mathrm{SU}(3)_{C}$ are equal, and $M_{1-2}$ the one where the coupling constants of $\mathrm{SU}(2)_{L}$ and $\mathrm{U}(1)_{Y}$ are equal. In particular, the matching scale is lower than the scale $M_{1-2}$, i.e., the conventional definition of the unification scale, in both models. Thus, the decay rate of the proton is higher than the conventional estimate, which uses $M_{1-2}$ $\sim 2 \times 10^{16} \mathrm{GeV}$ as the GUT gauge-boson mass.

\section{GAUGE-BOSON MASS IN THE SU(5) ${ }_{\mathrm{GUT}} \times \mathrm{U}(2)_{H}$ MODEL}

Let us now proceed from the discussion at the tree level to the next-to-leading-order analysis in order to give more precise predictions. To find the proton-decay rate, it is necessary to determine the mass of the gauge boson, rather than the matching scale. The GUT gauge-boson mass enters the threshold corrections to the gauge-coupling constants at the one-loop level and hence it can be discussed directly.

The analysis in this article follows the procedure described in [23]. First, the three gauge-coupling constants of the MSSM are given in terms of the gauge-coupling constants and other parameters of GUT models. We include one- 
TABLE III. Summary of the particle spectrum around the GUT scale of the $\mathrm{SU}(5)_{\mathrm{GUT}} \times \mathrm{U}(2)_{H}$ model. The first line denotes the representation under the gauge group of the MSSM. In the second line, "m.vect." denotes $\mathcal{N}=1$ massive vector multiplets and $\chi+\chi^{\dagger}$ a pair of $\mathcal{N}=1$ chiral and antichiral multiplets. In the last line, the mass of each multiplet is given in terms of gauge-coupling constants and parameters in the superpotential (3).

\begin{tabular}{lcccc}
\hline \hline$(\mathbf{3}, \mathbf{2})^{-5 / 6}$ & $(\mathbf{1}, \mathbf{1})^{0}$ & $(\mathbf{1}, \mathbf{1})^{0}$ & $(\mathbf{1}, \mathbf{a d j})^{0}$ & $(\mathbf{1}, \mathbf{a d j})^{0}$ \\
m.vect. & m.vect. & $\chi+\chi^{\dagger}$ & m.vect. & $\chi+\chi^{\dagger}$ \\
$M_{G}=\sqrt{2} g_{\mathrm{GUT}} v$ & $M_{1 V}=\sqrt{2\left(g_{1 H}^{2}+3 g_{\mathrm{GUT}}^{2} / 5\right)} v$ & $M_{1 C}=\sqrt{2} \lambda_{1 H} v$ & $M_{3 V}=\sqrt{2\left(g_{2 H}^{2}+g_{\mathrm{GUT}}^{2}\right)} v$ & $M_{3 C}=\sqrt{2} \lambda_{2 H}$ \\
\hline \hline
\end{tabular}

loop threshold corrections from the GUT-scale spectra to the matching equations. Then, we constrain the parameters of the GUT models by the three matching equations: three parameters are determined, and the other parameters are left undetermined. The free parameters, however, cannot be completely free when we require that the GUT models be in a calculable regime, i.e., when a perturbation analysis is valid. We determine the calculable region in the space of the free parameters and, as a result, the ranges of the GUT gaugeboson masses are obtained for the models.

In the minimal SU(5) SUSY GUT model, for example, there are four parameters. The three gauge-coupling constants of the MSSM determine the mass of the colored Higgs multiplets and put two independent constraints between the other three parameters. The three parameters are the unified gauge-coupling constant, the GUT gauge-boson mass, and a coefficient of the cubic coupling of the SU(5) $)_{\mathrm{GUT}}$-adj chiral multiplet in the superpotential [23]. Thus, the matching equations cannot determine the GUT gauge-boson mass directly. The cubic coupling coefficient is chosen as the free parameter, while the GUT gauge-boson mass and the unified gauge-coupling constant are solved in terms of the free parameter and the MSSM gauge-coupling constants. The free parameter, however, cannot be too large; otherwise it would immediately make itself extremely large in the renormalization-group evolution toward the ultraviolet (UV). Thus, it is bounded from above, and its upper bound leads to the lower bound of the GUT gauge-boson mass of the minimal SU(5) model [23].

The $\mathrm{SU}(5)_{\mathrm{GUT}} \times \mathrm{U}(N)_{H}$ model $[N=2$ (or 3)] has five (or six) parameters in the three matching equations of gauge coupling constants, as we see later. Thus, two (or three) parameters are left undetermined. The space of two (or three) free parameters is restricted by requiring the perturbation analysis to be valid, just as in the analysis of the minimal SU(5) model. As a result, the range of the GUT gauge-boson mass is obtained. The crucial difference between the three models is that only the lower bound of the mass is obtained in the minimal SU(5) model, while the upper bound is obtained both in the $\mathrm{SU}(5)_{\mathrm{GUT}} \times \mathrm{U}(2)_{H}$ model and in the $\mathrm{SU}(5)_{\mathrm{GUT}} \times \mathrm{U}(3)_{H}$ model, ${ }^{9}$ as shown in the following. The $\mathrm{SU}(5)_{\mathrm{GUT}} \times \mathrm{U}(2)_{H}$ model is analyzed in this section, and the result of the $\mathrm{SU}(5)_{\mathrm{GUT}} \times \mathrm{U}(3)_{H}$ model is described in Sec. IV.

${ }^{9}$ The lower bound also exists in this model.

\section{A. Parameters of the model}

The MSSM gauge-coupling constants are given in terms of parameters of the $\mathrm{SU}(5)_{\mathrm{GUT}} \times \mathrm{U}(2)_{H}$ model at the oneloop level as

$$
\begin{aligned}
\frac{1}{\alpha_{3}}(\mu)= & \frac{1}{\alpha_{\mathrm{GUT}}}(M)+\frac{3}{2 \pi} \ln \left(\frac{\mu}{M}\right)+\frac{4}{2 \pi} \ln \left(\frac{M_{G}}{M}\right), \\
\frac{1}{\alpha_{2}}(\mu)= & \frac{1}{\alpha_{\mathrm{GUT}}}(M)+\frac{1}{\alpha_{2 H}}(M)+\frac{-1}{2 \pi} \ln \left(\frac{\mu}{M}\right) \\
& +\frac{6}{2 \pi} \ln \left(\frac{M_{G}}{M}\right)+\frac{4}{2 \pi} \ln \left(\frac{M_{3 V}}{M_{3 C}}\right), \\
\frac{1}{\alpha_{1}}(\mu)= & \frac{1}{\alpha_{\mathrm{GUT}}}(M)+\frac{3 / 5}{\alpha_{1 H}}(M)+\frac{-33 / 5}{2 \pi} \ln \left(\frac{\mu}{M}\right) \\
& +\frac{10}{2 \pi} \ln \left(\frac{M_{G}}{M}\right),
\end{aligned}
$$

where $M$ and $\mu$ are the renormalization points of the GUT model and the MSSM, respectively. The renormalization point $M$ is chosen to be above the GUT scale and $\mu$ to be just below the GUT scale. The right-hand sides consist of the tree-level contributions (the first and second terms) in Eqs. (4)-(6) and one-loop renormalization and threshold corrections (the remaining terms). The gauge-coupling constants are considered to be defined in the dimensional reduction $(\overline{\mathrm{DR}})$ scheme, and hence the step-function approximation is valid in the one-loop threshold corrections [25]. Various mass parameters of the model enter the equations through the threshold corrections; $M_{G}$ is the GUT gauge-boson mass; $M_{3 V}$ and $M_{3 C}$ are masses of the $\mathrm{SU}(2)_{L}$-adj vector multiplet and chiral multiplet, respectively. These mass parameters are given in terms of the parameters of the Lagrangian (at tree level), as shown in Table III.

There are five parameters of the GUT model in the above three equations: $M_{G}, M_{3 V} / M_{3 C}, 1 / \alpha_{2 H}, 1 / \alpha_{1 H}$, and $1 / \alpha_{\text {GUT }}$. Three of them are solved in terms of the other two parameters and of the three MSSM gauge-coupling constants. The other two parameters are left undetermined for the moment. We take $M_{G}$ and $M_{3 V} / M_{3 C}$ as the two independent free parameters. Then three others, namely, $\alpha_{\mathrm{GUT}}\left(M_{G}\right)$, $\alpha_{2 H}\left(M_{G}\right)$, and $\alpha_{1 H}\left(M_{G}\right)$, are determined through Eqs. (11)-(13) by setting $\mu=M=M_{G}$. Another parameter of the 

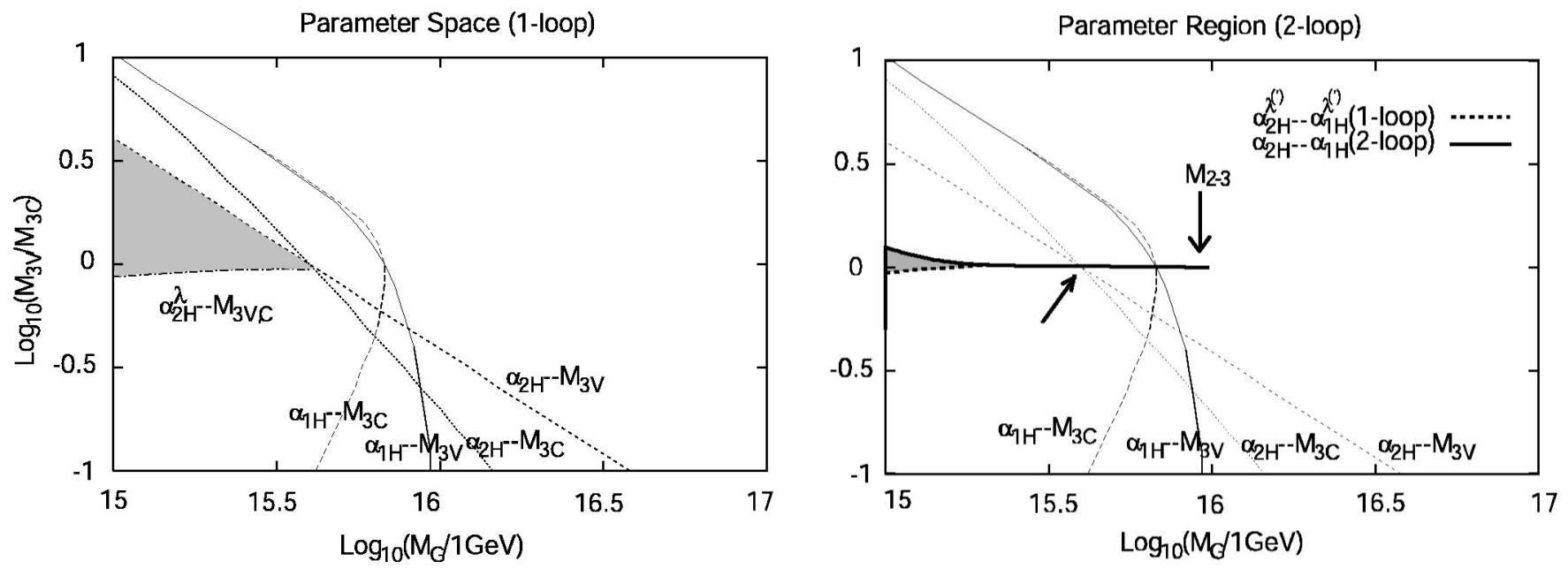

FIG. 2. Parameter region of the $\mathrm{SU}(5)_{\mathrm{GUT}} \times \mathrm{U}(2)_{H}$ model. The parameter space of the model spanned by two free parameters $M_{G}$ and $M_{3 V} / M_{3 C}$ is restricted by requiring that all the running coupling constants of the model remain finite while the renormalization point is below the heaviest particle of the model. The left panel shows the parameter region where the one-loop renormalization group is used for all the coupling constants. The right-hand sides of the four curves labeled "(gauge-coupling)-mass" are excluded. The region below the curve labeled " $\alpha_{2 \mathrm{H}^{\lambda}}^{\lambda} M_{3 V, C}$ " is also excluded. Thus, the parameter space of the model is restricted to the shaded triangular region. The right panel shows the parameter region (shaded) where two-loop effects are included in the renormalization-group equations of gauge-coupling constants. The four curves are those found in the left panel; we keep them just because they make it easier to compare the panel with the left one. The majority of the triangular region in the left panel is further excluded because of the two-loop effects, and only a small region survives near the line $M_{3 V} \simeq M_{3 C}$. The upper bound of $M_{G}$ is indicated by an arrow. In the right panel, $M_{2-3}$ indicates the unification point between $1 / \alpha_{2}$ and $1 / \alpha_{3}(+2 \sigma)$ (see Fig. 1 for details). It is easy to see that $M_{G} \lesssim 10^{15.6} \mathrm{GeV} \simeq\left(10^{-0.4} \simeq 0.40\right) \times\left(M_{2-3} \simeq 10^{16.0} \mathrm{GeV}\right)$. Both panels use $\alpha_{s}^{\overline{\mathrm{MS}},(5)}\left(M_{Z}\right)=0.1212$. The effects from the nonrenormalizable operator (22) are not included here.

model, $\alpha_{2 H}^{\lambda} \equiv\left(\lambda_{2 H}\right)^{2} /(4 \pi)$, is also expressed in terms of $\alpha_{2 H}\left(M_{G}\right), \alpha_{\mathrm{GUT}}\left(M_{G}\right)$, and $M_{3 V} / M_{3 C}$ :

$$
\frac{1}{\alpha_{2 H}^{\lambda}}\left(M_{G}\right)=\left(\frac{1}{\alpha_{2 H}\left(M_{G}\right)+\alpha_{\mathrm{GUT}}\left(M_{G}\right)}\right)\left(\frac{M_{3 V}}{M_{3 C}}\right)^{2} .
$$

\section{B. Parameter region of the model}

Let us now determine the parameter region in the parameter space spanned by $M_{G}$ and $M_{3 V} / M_{3 C}$. We require that the perturbation analysis be valid; it is necessary that all the coupling constants in the model be finite in the renormalization-group evolution toward the UV, at least within the range of the spectrum of the model. To be more explicit, the coupling constants $\alpha_{2 H}(M), \alpha_{2 H}^{\lambda}(M), \alpha_{2 H}^{\lambda^{\prime}}(M)$, $\alpha_{1 H}(M), \alpha_{1 H}^{\lambda}(M)$, and $\alpha_{1 H}^{\lambda^{\prime}}(M)$ are required to be finite in the renormalization-group evolution, at least while the renormalization point $M$ is below the heaviest particle of the model. We use this necessary condition to determine the parameter region. Here and hereafter, we adopt the following notation:

$$
\begin{gathered}
\alpha_{2 H}^{\lambda} \equiv \frac{\left(\lambda_{2 H}\right)^{2}}{4 \pi}, \quad \alpha_{2 H}^{\lambda^{\prime}} \equiv \frac{\left(\lambda_{2 H}^{\prime}\right)^{2}}{4 \pi}, \\
\alpha_{1 H}^{\lambda} \equiv \frac{\left(\lambda_{1 H}\right)^{2}}{4 \pi}, \quad \alpha_{1 H}^{\lambda^{\prime}} \equiv \frac{\left(\lambda_{1 H}^{\prime}\right)^{2}}{4 \pi} .
\end{gathered}
$$

First, we use the one-loop renormalization-group equation ${ }^{10}$ to determine the parameter region. The renormalization-group equations of this model are listed in Appendix A. The result is shown in the left panel of Fig. 2. The parameter region is given by the shaded region in the $M_{G}-\left(M_{3 V} / M_{3 C}\right)$ plane. The analysis is based on the value of $\alpha_{3}(\mu)$ calculated from $\alpha_{s}^{\overline{\mathrm{MS}},(5)}\left(M_{Z}\right)=0.1212$, i.e., the value larger than the central value by $2 \sigma$. The reason for this choice is explained shortly.

The result in the left panel is understood intuitively as follows. First, the parameter $M_{G}$ is bounded from above (from the right of the panel). This is a quite natural consequence, since it is consistent with the rough estimate of the matching scale in Sec. II C. Second, the parameter region is also bounded from below. This is also a natural consequence for the following reason. The beta function of the superpotential coupling $\alpha_{2 H}^{\lambda}$ in Eq. (A2) implies that this coupling constant immediately becomes large unless its contribution to the beta function is canceled by those from gauge interactions. Thus, the parameter space with $\alpha_{2 H} \ll \alpha_{2 H}^{\lambda}$, which is almost equivalent to $M_{3 V} \ll M_{3 C}$, is excluded.

\footnotetext{
${ }^{10}$ The beta function of $\alpha_{2 H}^{\lambda}(M)$ depends also on $\alpha_{2 H}^{\lambda^{\prime}}(M)$, $\alpha_{1 H}^{\lambda}(M)$, and $\alpha_{1 H}^{\lambda^{\prime}}(M)$. Thus, the beta function cannot be calculated without the values of those coupling constants. Their values, however, are not determined through the one-loop matching equations (11)-(13). Therefore, we set them in the beta function as 0 , so that $\alpha_{2 H}^{\lambda}(M)$ becomes large as slowly as possible in the evolution to the UV. This makes the excluded parameter space smaller and makes our analysis more conservative.
} 
We adopt the value $\alpha_{s}^{\overline{\mathrm{MS}},(5)}\left(M_{Z}\right)=0.1212$ for the value of the QCD coupling constant, rather than the usual central value $\alpha_{s}^{\overline{\mathrm{MS}},(5)}\left(M_{Z}\right)=0.1172$, because this allows $M_{2-3}$ to be larger. In turn, this allows the excluded region to be smaller, and hence the upper bound for the GUT gauge-boson mass becomes more conservative with this choice.

We further include two-loop effects in the beta functions of the gauge-coupling constants. ${ }^{11}$ The renormalizationgroup equations for the gauge-coupling constants are listed in Appendix A. The two-loop effects also become important at generic points of the parameter space, because the oneloop beta functions of the gauge coupling constants are accidentally small everywhere in the parameter space. ${ }^{12}$ The initial values of the two-loop renormalization-group evolution, i.e., the values at the matching scale $M=M_{G}$, are not determined for $\alpha_{2 H}^{\lambda^{\prime}}, \alpha_{1 H}^{\lambda}$, and $\alpha_{1 H}^{\lambda^{\prime}}$ from the matching equations (11)-(13). Thus, we set their values as

$$
\alpha_{2 H}^{\lambda^{\prime}}=\alpha_{2 H}^{\lambda}, \quad \alpha_{1 H}^{\lambda}=\alpha_{1 H}^{\lambda^{\prime}}=\alpha_{1 H},
$$

when the renormalization point $M$ is at $M_{G}$. Although we should have varied these values also as free parameters, we believe that the result of our analysis is not affected very much by changing these values; the reason is explained in Appendix B. The right panel of Fig. 2 is the parameter region determined in this analysis.

The right panel of Fig. 2 shows that the parameter space with $M_{3 V} \gg M_{3 C}$, i.e., $\alpha_{2 H} \gg \alpha_{2 H}^{\lambda}$, is further excluded, and the only surviving parameter region is around the line of $M_{3 V} \simeq M_{3 C}$, i.e., $\alpha_{2 H} \simeq \alpha_{2 H}^{\lambda}$. It is clear, as shown below, why this region and only this region survives. Let us neglect, for the moment, the renormalization effects from the $\mathrm{SU}(5)_{\text {GUT }}$ gauge interaction; the $\mathrm{SU}(5)_{\text {GUT }}$ gauge-coupling constant is smaller than those of the $\mathrm{SU}(2)_{H}$ and the $\mathrm{U}(1)_{H}$ interactions. Then, one can see that the two-loop parts of the beta functions of $\alpha_{2 H}$ and $\alpha_{1 H}$ are proportional to $\left(\alpha_{2 H}\right.$ $\left.-\alpha_{2 H}^{\lambda^{(\prime)}}\right)$ and $\left(\alpha_{1 H}-\alpha_{1 H}^{\lambda^{\left({ }^{\prime}\right)}}\right)$. Thus, the renormalization effects from $\alpha_{2 H}$ and $\alpha_{1 H}$ are completely canceled ${ }^{13}$ by $\alpha_{2 H}^{\lambda^{\left({ }^{\prime}\right)}}$ and $\alpha_{1 H}^{\lambda^{\left({ }^{\prime}\right)}}$ just in that region.

The cancellation in the two-loop beta functions is due to the $\mathcal{N}=2$ SUSY structure in the GUT-symmetry-breaking sector $[8,17,21]$; the beta functions of the gauge-coupling constants vanish at two loops and higher in the perturbative expansion in the $\mathcal{N}=2$ SUSY gauge theories [26]. Therefore, the remaining region at the two-loop level survives even if higher-loop effects are included in the beta functions.

The renormalization-group evolution is determined by the one-loop beta functions on the $\mathcal{N}=2$ SUSY line $\alpha_{2 H} \simeq \alpha_{2 H}^{\lambda}$

\footnotetext{
${ }^{11}$ Note that the beta functions are scheme independent up to two loops for gauge-coupling constants, but only up to one loop for coupling constants in the superpotential.

${ }^{12}$ At one-loop order, the beta function of $\alpha_{2 H}$ is given as a result of cancellation between the vector loop $3 T_{G}=6$ and the chiral loop $-T_{R}=-8$.

${ }^{13}$ Here, we assume that Eqs. (16) are also satisfied.
}

[when the $\mathrm{SU}(5)_{\mathrm{GUT}}$ interaction is neglected]. Therefore, we consider that the point in the parameter space indicated by an arrow in the right panel of Fig. 2 gives a conservative upper bound of $M_{G}$. We also consider that the upper bound so obtained is a good approximation of the maximum value of $M_{G}$, although the parameter region becomes thinner and thinner as $M_{G}$ increases; see Appendix B for a detailed discussion. Theoretical uncertainties in this determination of the conservative upper bound of $M_{G}$ are discussed in Sec. III C. A related discussion is also found in Appendix B.

Now that we know that the upper bound is obtained on the $\mathcal{N}=2$ SUSY line, it is possible to obtain the upper bound of $M_{G}$ without numerical analysis. Indeed, the following two facts make the analysis very simple: $M_{G}$ is essentially the only free parameter on the line, and the one-loop renormalization-group evolution is a good approximation there.

The gauge-coupling constant $\alpha_{2 H}$ is given at $M_{G}$ by

$$
\frac{1}{\alpha_{2 H}\left(M_{G}\right)}=\frac{-4}{2 \pi} \ln \left(\frac{M_{G}}{M_{2-3}}\right)
$$

through the matching equations (11) and (12), where a threshold correction proportional to $\ln \left(M_{3 V} / M_{3 C}\right)$ is neglected owing to the $\mathcal{N}=2$ SUSY. Here, $M_{2-3}$ is defined so that $\alpha_{3}\left(M_{2-3}\right)=\alpha_{2}\left(M_{2-3}\right)$. The gauge-coupling constant $\alpha_{2 H}$ so determined should not be too large because

$$
\frac{1}{\alpha_{2 H}\left(M_{3 V}\right)} \simeq \frac{1}{\alpha_{2 H}\left(M_{G}\right)}-\frac{2}{2 \pi} \ln \left(\frac{M_{3 V}}{M_{G}}\right) \gtrsim 0 .
$$

It follows only from the inequality ${ }^{14}$ in (18) that $(2 \pi) / \alpha_{2 \mathrm{H}}\left(M_{G}\right) \geq 3.7$; note that $M_{3 V} / M_{G}$ can be expressed in terms of $\alpha_{2 H}$ and $\alpha_{\mathrm{GUT}}$. Thus, the upper bound of $M_{G}$ is given by

$$
M_{G} \simeq e^{-2 \pi / 4 \alpha_{2 H}\left(M_{G}\right)} M_{2-3} \lesssim e^{-3.7 / 4} M_{2-3} \simeq 0.40 \times M_{2-3} .
$$

\section{Uncertainties in the upper bound of the gauge-boson mass}

Here, we estimate uncertainties in our prediction of the upper bound of the GUT gauge-boson mass. Uncertainties arising from our analysis of the GUT model are discussed in this subsection, while uncertainties arising from low-energy physics are discussed in Sec. V.

First, we focus on the effects from the $\mathrm{SU}(5)_{\mathrm{GUT}}$ interaction. They have been neglected ${ }^{15}$ in the discussion of the previous subsection, but they do contribute to the two-loop beta functions; in addition, the higher-loop contributions from $\alpha_{2 H}$ and $\alpha_{2 H}^{\lambda}$ no longer cancel because the $\mathrm{SU}(5)_{\mathrm{GUT}}$ interaction does not preserve $\mathcal{N}=2$ SUSY. Thus, the renormalization-group evolution is changed and the determination of the upper bound is affected. The $\mathrm{SU}(5)_{\mathrm{GUT}}$ inter-

\footnotetext{
14" 3.7" is almost independent of the SUSY threshold corrections to the MSSM gauge-coupling constants.

${ }^{15}$ They are not neglected in the numerical analysis in Fig. 2.
} 
TABLE IV. Summary of the particle spectrum around the GUT scale of the $\mathrm{SU}(5)_{\mathrm{GUT}} \times \mathrm{U}(3)_{H}$ model. The $\mathrm{SU}(5)_{\mathrm{GUT}}$ gauge coupling constant $g_{\text {GUT }}$ is abbreviated as $g$ in this table. See the caption for Table III for the conventions in this table, replacing "superpotential (3)" by "superpotential (7)."

\begin{tabular}{lcccccc}
\hline \hline$(\mathbf{3}, \mathbf{2})^{-5 / 6}$ & $(\mathbf{3}, \mathbf{1})^{-1 / 3}$ & $(\mathbf{3}, \mathbf{1})^{-1 / 3}$ & $(\mathbf{1}, \mathbf{1})^{0}$ & $(\mathbf{1}, \mathbf{1})^{0}$ & $(\mathbf{a d j}, \mathbf{1})^{0}$ & $(\mathbf{a d j}, \mathbf{1})^{0}$ \\
m.vect. & $\chi+\chi^{\dagger}$ & $\chi+\chi^{\dagger}$ & m.vect. & $\chi^{+} \chi^{\dagger}$ & m.vect. & $\chi+\chi^{\dagger}$ \\
$M_{G}=\sqrt{2} g v$ & $M_{H_{c}}=h v$ & $M_{H_{c}^{-}}=h^{\prime} v$ & $M_{1 V}=\sqrt{2\left(g_{1 H}^{2}+2 g^{2} / 5\right)}$ & $M_{1 C}=\sqrt{2} \lambda_{1 H} v$ & $M_{8 V}=\sqrt{2\left(g_{3 H}^{2}+g^{2}\right)} v$ & $M_{8 C}=\sqrt{2} \lambda_{3 H} v$ \\
\hline \hline
\end{tabular}

action contributes to the beta function of $\alpha_{2 H}$ in Eq. (A1) by less than $10 \%$ of the one-loop contribution. ${ }^{16}$ Thus, the value of $\alpha_{2 H}\left(M_{G}\right)$ for the upper-bound value of $M_{G}$ is not changed by $10 \%$ [see Eq. (18)]. As a result, the upper bound of $M_{G}$ is not modified by a factor of more than $e^{(2 \pi) /\left[4 \alpha_{2 \mathrm{H}}\left(M_{G}\right)\right] \times( \pm 10 \%)} \sim 10^{ \pm 0.04}$.

Second, the perturbative expansion would not converge when the 't Hooft coupling $2 \alpha_{2 H} /(4 \pi)$ exceeds unity. It is impossible to extract any definite statement on the renormalization-group evolution when the perturbative expansion is not valid. However, most of the renormalizationgroup evolution is in the perturbative regime, i.e., $\left[4 \pi /\left(2 \alpha_{2 H}\right)\right] \gtrsim 1$, since we know that $\left[4 \pi /\left(2 \alpha_{2 H}\right)\right]\left(M_{G}\right)$ $\simeq 3.7$ for the upper-bound value of $M_{G}$. Thus, we consider that the perturbation analysis in the previous subsection is fairly reliable.

Third, nonperturbative contributions are also expected in the beta functions, and they might not be neglected since the gauge-coupling constants are relatively large in this model. They ${ }^{17}$ are expected to be of the form [27]

$$
\begin{aligned}
(1- & \left.\frac{T_{G}}{2 \pi} \alpha\right) \frac{\partial}{\partial \ln \mu}\left(\frac{1}{\alpha}(\mu)\right) \\
& =\frac{3 T_{G}-T_{R}}{2 \pi}+\sum_{n=1}^{\infty} c_{n}\left(g^{-2 T_{G}} e^{-2 \pi / \alpha}\right)^{n},
\end{aligned}
$$

where the $c_{n}$ 's are numerical factors of the order of unity. Each contribution comes from $n$-instantons. Here, we neglected perturbative and nonperturbative contributions through wave-function renormalizations of hypermultiplets. This is because hypermultiplets of $\mathcal{N}=2$ SUSY gauge theories are protected from any radiative corrections [28]. We see that the nonperturbative effects given above are not significant when the renormalization point is around the GUT scale, since

$$
\left(g_{2 H}\right)^{-4} e^{-2 \pi / \alpha_{2 H}} \simeq 5 \times 10^{-5} \ll 1 .
$$

So far, the analysis is based on a renormalizable theory. However, the Yukawa couplings of quarks and charged leptons are given by nonrenormalizable operators in Eq. (3). Another nonrenormalizable operator is also necessary to account for the fact that the Yukawa coupling constants of the strange quark and muon are not unified in a simple manner. Those operators, however, affect the gauge-coupling con-

\footnotetext{
${ }^{16}$ See Appendix B for more details.

${ }^{17}$ We thank Tohru Eguchi for bringing this issue to our attention.
}

stants through the renormalization group only at higher-loop levels. Moreover, they are not relevant to the renormalization-group flow (say, in the sense of the Wilsonian renormalization group) except near the cutoff scale $M_{*}$. These are the reasons why we neglected the effects of those operators.

There may be, however, a nonrenormalizable operator:

$$
W=2 \operatorname{tr}\left[\left(\frac{1}{4 g^{2}}+c \frac{\langle\bar{Q} Q\rangle}{M_{*}^{2}}\right) \mathcal{W}^{\alpha} \mathcal{W}_{\alpha}\right],
$$

which directly modifies the matching equations of the gaugecoupling constants at the tree level. Exactly the same analysis as in Secs. III A and III B tells us that the upper bound of $M_{G}$ given in Eq. (19) is modified ${ }^{18}$ to

$$
M_{G} \lesssim 0.40 \times 10^{-0.82 \times c} \times M_{2-3},
$$

as long as $c \gtrsim\left[-3.7 /(6 \pi)+14 / 9 \times\left(\epsilon_{g} / \alpha_{\mathrm{GUT}}\right)\right] / 1.2 \simeq-0.16$ $+1.3 \times\left(\epsilon_{g} / \alpha_{\mathrm{GUT}}\right) ; \quad$ here, $\quad \epsilon_{g} \equiv\left[\left(g_{3}-g_{1}\right) / g_{1}\right]\left(M_{1-2}\right) \simeq$ $-(0.03-0.01)$.

\section{GAUGE-BOSON MASS IN THE SU(5) $)_{\mathrm{GUT}} \times \mathrm{U}(3)_{H}$ MODEL}

\section{A. Parameter region of the model}

The same analysis as in Sec. III is performed for the $\mathrm{SU}(5)_{\mathrm{GUT}} \times \mathrm{U}(3)_{H}$ model. The one-loop matching equations in this model, which are quite similar to Eqs. (11)-(13), are found in [21]. The particle spectrum around the GUT scale, which comes into the threshold corrections, is summarized in Table IV.

There are six parameters in the matching equations: $M_{G}$, $M_{8 V} / M_{8 C}, 1 / \alpha_{\mathrm{GUT}}, 1 / \alpha_{3 H}$, and $1 / \alpha_{1 H}$, just as in the $\mathrm{SU}(5)_{\mathrm{GUT}} \times \mathrm{U}(2)_{H}$ model, and $M_{H_{c}} M_{H_{c}^{-}} / M_{G}^{2}$. Three of them are fixed through the matching equations, and the other three are left undetermined. We take $M_{G}, M_{8 V} / M_{8 C}$, and $M_{H_{c}} M_{H_{c}^{-}} / M_{G}^{2}$ as the three free parameters. The space of these parameter is restricted by requiring that all the coupling constants $\alpha_{3 H}(M), \alpha_{3 H}^{\lambda\left(^{\prime}\right)}, \alpha_{1 H}(M)$, and $\alpha_{1 H}^{\lambda\left(^{\prime}\right)}$ be finite in the renormalization-group evolution toward the UV, at least within the range of the spectrum.

The parameter region is shown in Fig. 3; only the $\sqrt{\left(M_{H_{c}} M_{H_{c}^{-}} / M_{G}^{2}\right)}=10^{0.3}$ cross section is described, and

\footnotetext{
${ }^{18}$ Contributions to Eqs. (11)-(13) are, for example, $c \times 16 \pi$ $\times 0.024 \simeq 1.2 \times c$ at the point in the parameter space indicated by an arrow in Fig. 2.
} 


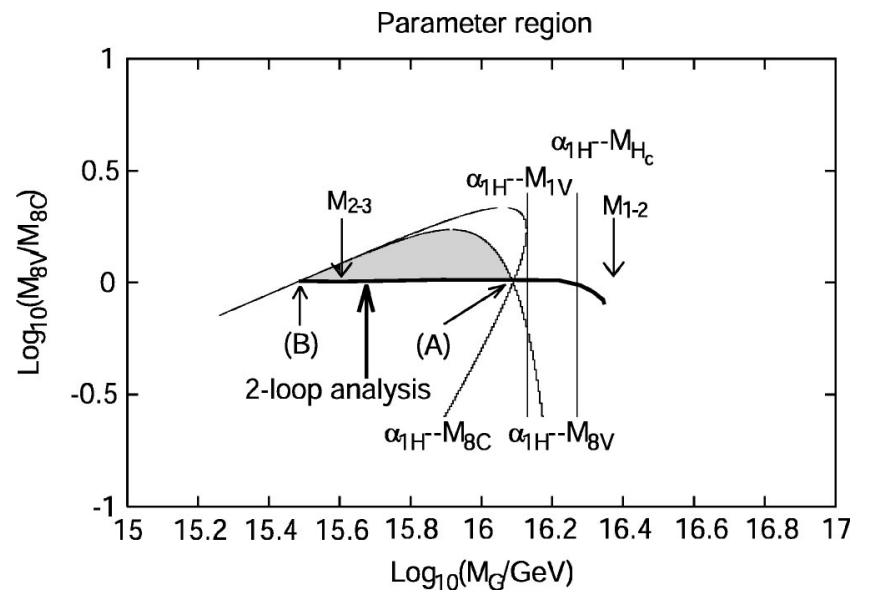

FIG. 3. Parameter region of the $\mathrm{SU}(5)_{\mathrm{GUT}} \times \mathrm{U}(3)_{H}$ model. The parameter space of the model is spanned by three independent parameters: $M_{G}, M_{8 V} / M_{8 C}$, and $\left(M_{H_{c}} M_{H_{c}^{-}}\right) / M_{G}^{2}$. The figure is the $\sqrt{\left(M_{H_{c}} M_{H_{c}^{-}}\right) / M_{G}^{2}}=10^{0.3}$ cross section of the parameter space. We require that all the coupling constants remain finite under the renormalization group, while the renormalization point is below the heaviest particle of the model. This condition is satisfied in the shaded region when the one-loop renormalization group is used. Thin curves and lines labeled "(gauge-coupling)-mass" are lines where the corresponding gauge-coupling constants become infinite at the corresponding mass scales. After two-loop effects are included in the beta functions of the gauge-coupling constants, the remaining allowed parameter region is only on the thick curve labeled two-loop analysis. Points (A) and (B) denote the upper and the lower bounds of the gauge-boson mass $M_{G}$, respectively, for fixed $\sqrt{\left(M_{H_{c}} M_{H_{c}^{-}}\right) / M_{G}^{2}}=10^{0.3}$. The upper bound of $M_{G}$ in the model is obtained as the maximum value $M_{G}$ takes at (A) as $\sqrt{\left(M_{H_{c}} M_{H_{c}^{-}}\right) / M_{G}^{2}}$ changes. One also sees immediately that the lower bound at (B) is so low that it is of no physical importance. $M_{1-2}\left[M_{2-3}\right]$ indicates the unification point between $1 / \alpha_{1}$ and $1 / \alpha_{2}\left[1 / \alpha_{2}\right.$ and $\left.1 / \alpha_{3}(-2 \sigma)\right]$ (see Fig. 1 for more details). Note that $\left[M_{G}\right.$ at $\left.(\mathrm{A})\right] \quad<10^{16.13} \mathrm{GeV} \simeq\left[\left(0.60 \simeq 10^{-0.22}\right) \times\left(M_{1-2}\right.\right.$ $\left.\left.\simeq 10^{16.35} \mathrm{GeV}\right)\right]$. The QCD coupling constant $\alpha_{s}^{\overline{\mathrm{MS}},(5)}\left(M_{Z}\right)$ $=0.1132$ is used. The effects from a nonrenormalizable operator that corresponds to Eq. (22) in this model are not included here.

hence the region is described in the $M_{G^{-}}\left(M_{8 V} / M_{8 C}\right)$ plane. This analysis is based on the value of $\alpha_{3}(\mu)$ that is calculated from $\alpha_{s}^{\overline{\mathrm{MS}},(5)}\left(M_{Z}\right)=0.1132$, i.e., a value smaller than the central value by $2 \sigma$. This is because it makes our analysis more conservative. The parameter region obtained by the one-loop renormalization group is shown as the shaded area in Fig. 3. The region is bounded from the right and from the left, which is again consistent with the rough estimate of the matching scale given in Sec. II C. The region is also bounded from below just for the same reason as in Sec. III B. The parameter region distant from the $\mathcal{N}=2$ SUSY line ${ }^{19} M_{8 V}$

\footnotetext{
${ }^{19}$ The $\mathcal{N}=2$ SUSY is enhanced in the GUT-symmetry-breaking sector when

$$
g_{1 \mathrm{H}} \simeq \lambda_{1 \mathrm{H}}\left(\sim \lambda_{1 \mathrm{H}}^{\prime}\right), \quad g_{3 \mathrm{H}} \simeq \lambda_{3 \mathrm{H}}\left(\sim \lambda_{3 \mathrm{H}}^{\prime}\right)
$$

$\simeq M_{8 C}$ is excluded when two-loop effects are included ${ }^{20}$ in the beta functions of the gauge-coupling constants; the twoloop contributions have significant effects compared with one-loop effects, because the one-loop beta function of $\alpha_{3 H}$ accidentally vanishes. The one-loop renormalization-group evolution is reliable on the $\mathcal{N}=2$ SUSY line, the thick line in Fig. 3, and hence the points indicated by (A) and (B) give the upper and lower bounds of $M_{G}$, respectively, in the $\sqrt{\left(M_{H_{c}} M_{H_{c}^{-}} / M_{G}^{2}\right)}=10^{0.3}$ cross section. The upper and lower bounds of $M_{G}$ of the model are the maximum and minimum values that $M_{G}$ takes at (A) and (B), respectively, when the remaining parameter $\left(M_{H_{c}} M_{H_{c}^{-}} / M_{G}^{2}\right)$ is varied. Since it is evident from the figure that the lower bound of $M_{G}$ leads to too fast a proton decay, we focus in the following only on the upper bound of $M_{G}$.

\section{B. Uncertainties in the upper bound of the gauge-boson mass}

In this subsection, we estimate uncertainties in the prediction of the upper bound of the GUT gauge-boson mass obtained in the previous subsection. The uncertainties that originate from low-energy physics, however, are discussed in Sec. V.

First, we discuss the effects of the interactions that violate $\mathcal{N}=2$ SUSY. The $\mathrm{SU}(5)_{\text {GUT }}$ gauge interaction and the cubic couplings in the fourth line of Eq. (7) are the sources of the violation of the $\mathcal{N}=2$ SUSY. Those interactions change the one-loop-exact evolution of $\mathcal{N}=2$ SUSY gauge theories. The change in the upper bound of $M_{G}$ comes ${ }^{21}$ from the change in the evolution of $\alpha_{1 H}$, because the upper bound was determined by the running of $\alpha_{1 H}$ in the absence of $\mathcal{N}=2$ SUSY breaking. The beta function of $\alpha_{1 H}$ is changed at most by a few percent, ${ }^{22}$ which leads to a change of the upper bound of $M_{G}$ by a factor of at most $10^{ \pm 0.01}$.

Second, one can see from the matching equations [21] of this model that the gauge-coupling constants $\alpha_{3 H}$ and $\alpha_{1 H}$ are not so large as to invalidate the perturbative expansion when the colored Higgs particles are moderately heavier than the GUT gauge-boson mass; only one threshold correction from the colored Higgs particles is sufficient to keep both coupling constants within the perturbative regime. Nonperturbative effects are not important at all in such a region.

Finally, a nonrenormalizable operator that corresponds to Eq. (22) may also exist in this model. Such an operator, if it

\footnotetext{
${ }^{20}$ We set the initial values $\left(M=M_{G}\right)$ of the coupling constants that are not determined by the matching equation as follows:

$$
h=h^{\prime}, \quad \alpha_{3 H}^{\lambda}=\alpha_{3 H}^{\lambda^{\prime}}, \quad \alpha_{1 H}^{\lambda}=\alpha_{1 H}^{\lambda^{\prime}}=\alpha_{1 H} .
$$

This choice makes the renormalization-group evolution the most stable.

${ }^{21}$ We confirmed that the change in the evolution of $\alpha_{3 H}$ is not so significant as to make the finiteness of $\alpha_{3 H}$ a more severe condition than that of $\alpha_{1 H}$.

${ }^{22}$ This estimate comes from the ratio between the one-loop contribution and the $\mathrm{SU}(5)_{\mathrm{GUT}}$ contribution at two loops. Note also that the $\alpha_{h} \equiv h^{2} /(4 \pi)$ contribution has a sign opposite to that of the $\mathrm{SU}(5)_{\mathrm{GUT}}$ contribution.
} 
exists, contributes to the matching equations at the tree level. In its presence, we can perform exactly the same analysis as in the previous subsection. The result of this analysis is presented in Sec. VI.

\section{CONSERVATIVE UPPER BOUND OF PROTON LIFETIME}

The analysis in Secs. III and IV presented the way of extracting the upper bound of the GUT gauge-boson mass for both models. The lifetime of the proton through GUT gauge-boson exchange is given [23] in terms of $M_{G}$ as

$$
\begin{aligned}
\tau\left(p \rightarrow \pi^{0} e^{+}\right) \simeq & 1.0 \times 10^{35} \times\left(\frac{0.015 \mathrm{GeV}^{3}}{\alpha_{H}}\right)^{2}\left(\frac{2.5}{A_{R}}\right)^{2} \\
& \times\left(\frac{1}{25 \alpha_{\mathrm{GUT}}\left(M_{G}\right)}\right)^{2}\left(\frac{M_{G}}{10^{16} \mathrm{GeV}}\right)^{4} \mathrm{yr},
\end{aligned}
$$

where $\alpha_{H}$ is a hadron matrix element ${ }^{23}$ calculated with lattice quenched QCD [30] $\left(\alpha_{H}=-0.015 \pm 0.001 \mathrm{GeV}^{3}\right)$ renormalized at $2.3 \mathrm{GeV}$, and $A_{R} \simeq 2.5$ is a renormalization factor of the dimension- 6 proton-decay operators [31]; $A_{R}$ consists of a short-distance part $A_{R}^{(\mathrm{SD})} \simeq 2.1$, which comes from the renormalization between the GUT scale and the electroweak scale, and a long-distance part ${ }^{24} A_{R}^{(\mathrm{LD})} \simeq 1.2$, from the renormalization between the electroweak scale and $2.3 \mathrm{GeV}\left(A_{R}\right.$ $=A_{R}^{(\mathrm{SD})} \cdot A_{R}^{(\mathrm{LD})}$ ). We note the expression of $A_{R}^{(\mathrm{SD})}$ [32] for later convenience:

$$
\begin{aligned}
A_{R}^{(\mathrm{SD})}= & \left(\frac{\alpha_{C}\left(M_{Z}\right)}{\alpha_{C}\left(M_{G}\right)}\right)^{4 / 3 /\left(b_{3}=3\right)}\left(\frac{\alpha_{L}\left(M_{Z}\right)}{\alpha_{L}\left(M_{G}\right)}\right)^{3 / 2 /\left(b_{2}=-1\right)} \\
& \times\left(\frac{\alpha_{Y}\left(M_{Z}\right)}{\alpha_{Y}\left(M_{G}\right)}\right)^{23 / 30 /\left(b_{1}=-33 / 5\right)},
\end{aligned}
$$

where $b_{i}(i=1,2,3)$ are coefficients of the one-loop beta functions of the three gauge-coupling constants of the MSSM. The renormalization from Yukawa coupling constants is omitted because its effect is negligible.

${ }^{23}$ The hadron matrix element $\alpha_{H}$ is defined by $\left\langle\operatorname{vac}\left|\left(u_{R} d_{R}\right) u_{L}\right| p(\overrightarrow{\mathbf{k}})\right\rangle=\alpha_{\mathrm{H}} u(\overrightarrow{\mathbf{k}})$. This is related to another matrix element $W\left(\simeq-0.15 \pm 0.02 \mathrm{GeV}^{2}\right)$ through

$$
\frac{\alpha_{\mathrm{H}}}{\sqrt{2} f_{\pi}}=-W
$$

where $W$ is defined by $\lim _{\overrightarrow{\mathbf{p}} \rightarrow \mathbf{0}}\left\langle\pi^{0}(\overrightarrow{\mathbf{p}})\left|\left(u_{R} d_{R}\right) u_{L}\right| p(\overrightarrow{\mathbf{k}})\right\rangle=W u(\overrightarrow{\mathbf{k}})$, and $f_{\pi}$ is the pion decay constant $\left(2 f_{\pi}=130 \pm 5 \mathrm{MeV}\right.$ [29]).

${ }^{24}$ The numerical coefficient of the formula of the lifetime adopted in [21] is different from the one in Eq. (26) in this article. This is because the formula in [21] is based implicitly on $A_{R} \simeq 3.6$ in [32], whose value is the effect of renormalization between the GUT scale and $1 \mathrm{GeV}$. It was therefore incorrect, in [21], to use at the same time $A_{R} \simeq 3.6$ renormalized at $1 \mathrm{GeV}$ and the hadron matrix element in [30] renormalized at $2.3 \mathrm{GeV}$.
Threshold corrections from SUSY particles are of the same order as those from the particles around the GUT scale. The two-loop effects in the renormalization-group evolution between the electroweak scale and the GUT scale are also of the same order. Therefore, the above two effects should be taken into consideration in deriving predictions for the GUT gauge-boson mass (and hence for the lifetime of the proton). This implies, in particular, that the predictions depend on the spectrum of SUSY particles. We present the predictions for the upper bound of the lifetime of the proton as a function of SUSY-breaking parameters of the minimal supergravity (MSUGRA) boundary condition in Sec. V A. Predictions can be obtained also for other SUSY-particle spectra such as that of gauge-mediated SUSY breaking (Sec. V B). Section V C discusses how the predictions are changed when there are vectorlike $\mathrm{SU}(5)_{\mathrm{GUT}}$ multiplets at a scale below the GUT scale.

\section{A. MSUGRA SUSY threshold corrections}

Let us first consider the SUSY-particle spectrum determined by the MSUGRA boundary condition. This spectrum and the MSSM gauge-coupling constants in the $\overline{\mathrm{DR}}$ scheme are calculated in an iterative procedure. We use the SOFTSUSY1.7 code [33] for this purpose. These coupling constants are evolved up to the GUT scale through the two-loop renormalization group. They are used as input in the left-hand sides of, say, Eqs. (11)-(13), to obtain a prediction of the upper bound of the GUT gauge-boson mass. The universal scalar mass $m_{0}$ and the universal gaugino mass $m_{1 / 2}$ are varied, while we fix the other parameters of the MSUGRA boundary condition ${ }^{25}$ as $\tan \beta=10.0, A_{0}=0 \mathrm{GeV}$, and the sign of the $\mu$ parameter is the standard one.

The left panel of Fig. 4 is a contour plot on the $m_{0}-m_{1 / 2}$ plane, describing the upper bound of the proton lifetime in the $\mathrm{SU}(5)_{\mathrm{GUT}} \times \mathrm{U}(2)_{H}$ model, where we set the unknown coefficient $c$ of the nonrenormalizable operator (22) to zero. The QCD coupling constant $\alpha_{s}^{\overline{\mathrm{MS}},(5)}\left(M_{Z}\right)=0.1212$ is used, so that the upper bound becomes more conservative. One can see that this upper bound ranges over $(1.4-3.2) \times 10^{33} \mathrm{yr}$. Notice that the $\left(m_{0}, m_{1 / 2}\right)$ dependence of the proton lifetime arises almost only through the variation of $M_{2-3}$ [see Eq. (19)]. Indeed, the contours of the upper bound of the lifetime in the left panel of Fig. 4 behave in the same way as those of $M_{2-3}$ in the upper left panel of Fig. 5.

It is now easy to see how much the prediction is changed when we adopt the central value of the QCD coupling constant, $\alpha_{s}^{\overline{\mathrm{MS}},(5)}\left(M_{Z}\right)=0.1172$. Since the choice of the QCD coupling constant directly changes $M_{2-3}$, it severely affects the upper bound of $M_{G}$ in this model. $M_{2-3}$ is decreased by a factor $e^{-\left[2 \pi /\left(b_{3}-b_{2}\right)\right][(1 / 0.1172)-(1 / 0.1212)]}$, and the lifetime is shortened by a factor of $e^{-2 \pi \times 0.28} \simeq 0.2$. We confirmed that the upper bound of the lifetime does not exceed $1 \times 10^{33} \mathrm{yr}$ even when the SUSY-breaking parameters $m_{0}$ and $m_{1 / 2}$ are

\footnotetext{
${ }^{25}$ This is because changes in these parameters did not change the result at all as in [21].
} 

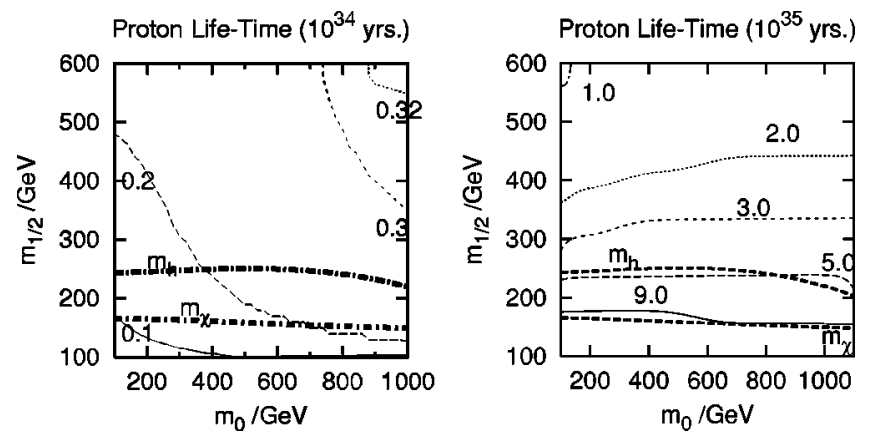

FIG. 4. Contour plots of the upper bound of the proton lifetime on the MSUGRA parameter space. The left panel is the prediction of the $\mathrm{SU}(5)_{\mathrm{GUT}} \times \mathrm{U}(2)_{H}$ model and the right one that of the $\mathrm{SU}(5)_{\mathrm{GUT}} \times \mathrm{U}(3)_{H}$ model. The upper bound changes as the universal scalar mass $m_{0}$ and the universal gaugino mass $m_{1 / 2}$ are varied (other MSUGRA parameters are fixed at $\tan \beta=10, A_{0}=0.0$ ). The $\mu$ parameter is chosen to be positive, when the constraint from the branching ratio of the $b \rightarrow s \gamma$ process is less severe. The upper bound of the lifetimes varies as $(1.4-3.2) \times 10^{33} \mathrm{yr}$ in the $\mathrm{SU}(5)_{\mathrm{GUT}} \times \mathrm{U}(2)_{H}$ model, where the QCD coupling constant $\alpha_{s}^{\overline{\mathrm{MS}},(5)}\left(M_{Z}\right)=0.1212$ is used. The upper bound varies as $(1-5)$ $\times 10^{35} \mathrm{yr}$ in the $\mathrm{SU}(5)_{\mathrm{GUT}} \times \mathrm{U}(3)_{H}$ model, where the QCD coupling constant $\alpha_{s}^{\overline{\mathrm{MS}},(5)}\left(M_{Z}\right)=0.1132$ is used. In both panels, the effects from nonrenormalizable operators are not included. The thick curves labeled $m_{h}$ and $m_{\chi}$ are the bounds on the MSUGRA parameter space from the LEP II experiment in search of the lightest Higgs boson ( $m_{h} \geqslant 114 \mathrm{GeV}, 95 \%$ C.L.) [40] and the lightest chargino ( $m_{\chi} \geqslant 103.5 \mathrm{GeV}, 95 \%$ C.L.) [41]. These curves are obtained by using the SOFTSUSY1.7 code [33]. The excluded region changes when other codes are used; lower bound of $m_{1 / 2}$ for fixed $m_{0}$ can be higher by about $100 \mathrm{GeV}$. The code we adopt yields the largest pole mass of the lightest Higgs scalar among various codes available [34], and hence the excluded region is the smallest.

varied up to $2000 \mathrm{GeV}$ if we adopt the central value of the QCD coupling constant.

The hadron matrix element $\alpha_{H}$ in [30], which has a statistical error $\alpha_{H}=-0.015 \pm 0.001 \mathrm{GeV}^{3}$, does not include a systematic error (e.g., an error due to the quenched approximation). Reference [35] estimates that the systematic error is about $50 \%$, which leads to an uncertainty in the lifetime of a factor of 2 .

Therefore, the conservative upper bound is roughly $\tau \lesssim 6$ $\times 10^{33} \mathrm{yr}$, where we exploit the uncertainties in the SUSY threshold corrections, in the value of the QCD coupling constant, and in the hadron matrix element. Thus, the prediction does not contradict the experimental lower bound from the Super-Kamiokande experiment, $\tau\left(p \rightarrow \pi^{0} e^{+}\right) \gtrsim 5 \times 10^{33} \mathrm{yr}$ (90\% C.L.) [36] at this moment, ${ }^{26}$ yet a large portion of the parameter region is already excluded. Moreover, one can expect that the uncertainties originating from low-energy physics will be reduced in the future. Thus, further accumulation of data in Super-Kamiokande and the next generation of water Cerenkov detectors will be sure either to exclude this

\footnotetext{
${ }^{26}$ The lifetime listed in [29] is $\tau\left(p \rightarrow \pi^{0} e^{+}\right) \geq 1.6 \times 10^{33} \mathrm{yr}(90 \%$ C.L.), based on a paper [37] published in 1998.
}
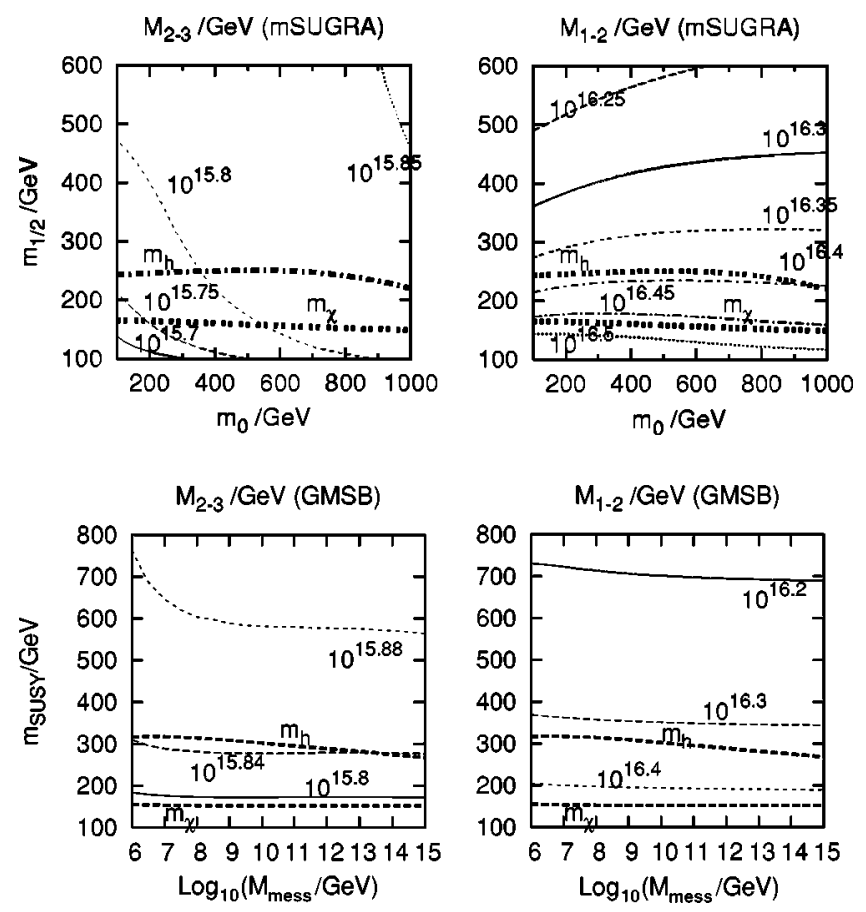

FIG. 5. The left panels show the contour plots of the energy scale $M_{2-3}$, where the $\mathrm{SU}(2)_{L}$ and $\mathrm{SU}(3)_{C}$ gauge-coupling constants become the same. The right panels show those of the energy scale $M_{1-2}$, where the $\mathrm{U}(1)_{Y}$ and $\mathrm{SU}(2)_{L}$ gauge-coupling constants become the same. The upper panels are contour plots on the $\left(m_{0}, m_{1 / 2}\right)$ parameter space of MSUGRA SUSY breaking, the lower ones are for the $\left(M_{\text {mess }}, m_{\mathrm{SUSY}} \equiv[(1 / 24) /(4 \pi)] \Lambda\right)$ parameter space of the GMSB. Other parameters are fixed for both SUSY breakings; $A_{0}=0 \mathrm{GeV}$ for MSUGRA SUSY breaking, and $\tan \beta=10.0$ and $\mu>0$ for both SUSY breakings. $\alpha_{s}^{\overline{\mathrm{MS}},(5)}\left(M_{Z}\right)=0.1172$ is used as the QCD coupling constant in this figure. See the caption for Fig. 4 for more details about the region excluded by the LEP II experiments.

model without the nonrenormalizable operator (22), or to detect the proton decay.

Now, we move to consider the $\mathrm{SU}(5)_{\mathrm{GUT}} \times \mathrm{U}(3)_{H}$ model. The right panel of Fig. 4 is a contour plot on the $m_{0}-m_{1 / 2}$ plane, describing the upper bound of the proton lifetime in the $\mathrm{SU}(5)_{\mathrm{GUT}} \times \mathrm{U}(3)_{H}$ model. The QCD coupling constant $\alpha_{s}^{\overline{\mathrm{MS}},(5)}\left(M_{Z}\right)=0.1132$ is used. The upper bound of the proton lifetime ranges over $(1-5) \times 10^{35} \mathrm{yr}$ on the MSUGRA parameter space that is not excluded by the CERN $e^{+} e^{-}$collider LEP II bound on the lightest-Higgs-boson mass.

Let us now see how much the above prediction is changed by uncertainties related to the QCD. First, the following observation is important in discussing the effect from the uncertainty in the value of the QCD coupling constant. The behavior of the contours of the upper bound of the lifetime, and hence of the GUT gauge-boson mass, has, in this model, strong correlations with that of $M_{1-2}$ presented in the upper right panel of Fig. 5. We find an empirical relation

$$
M_{G} \lesssim 0.60 \times M_{1-2} .
$$

Thus, the upper bound does not depend on the value of the QCD coupling constant very much, since $M_{1-2}$ is not af- 
fected very much. Second, the uncertainty in the hadron form factor is common to both models. Therefore, the most conservative upper bound of the proton lifetime is roughly $\tau$ $\$ 10^{36} \mathrm{yr}$ in this model. In particular, the proton decay might not be within the reach of the next generation of experiments.

\section{B. Threshold corrections from various SUSY-particle spectra}

Gauge-mediated SUSY breaking (GMSB) is one of the highly motivated models of SUSY breaking. The spectrum of the SUSY particles is different from that of the MSUGRA SUSY breaking, and, moreover, there are extra $\mathrm{SU}(5)_{\mathrm{GUT}}$ charged particles as messengers. Thus, the predictions for the proton lifetime are different from those in the case of MSUGRA SUSY breaking. We discuss the effects of the difference in the SUSY-particle spectra in this subsection. A possible change of predictions due to the existence of extra particles is discussed in the next subsection.

The ranges of the GUT gauge-boson masses are different for different SUSY-particle spectra, yet the difference arises only from the difference in the two energy scales $M_{2-3}$ and $M_{1-2}$ : the energy scale where the $\mathrm{SU}(2)_{L}$ and $\mathrm{SU}(3)_{C}$ coupling constants become the same and where those of $\mathrm{U}(1)_{Y}$ and $\mathrm{SU}(2)_{L}$ become the same, respectively. The upper bound of $M_{G}$ is given in terms of $M_{2-3}$ through Eq. (19) in the $\mathrm{SU}(5)_{\mathrm{GUT}} \times \mathrm{U}(2)_{H}$ model, and in terms of $M_{1-2}$ through Eq. (29) in the $\mathrm{SU}(5)_{\mathrm{GUT}} \times \mathrm{U}(3)_{H}$ model.

Figure 5 shows how $M_{2-3}$ and $M_{1-2}$ vary over the parameter space of GMSB. The parameter space is spanned by two parameters: an overall mass scale $\Lambda$ of the SUSY breaking in the MSSM sector and the messenger mass $M_{\text {mess }}$. We assume that the messenger sector consists of one pair of $\mathrm{SU}(5)_{\mathrm{GUT}^{-}}\left(\mathbf{5}+\mathbf{5}^{*}\right)$ representations. The gaugino masses are given by

$$
m_{\tilde{g}_{i}}=\frac{\alpha_{i}}{4 \pi} \Lambda\left[1+\mathcal{O}\left(\frac{\Lambda}{M_{\text {mess }}}\right)\right] \quad(i=1,2,3)
$$

at the threshold $M_{\text {mess }}$. We calculate the SUSY-particle spectrum, the SUSY threshold corrections to the MSSM gaugecoupling constants, and the renormalization-group evolution to the messenger scale using the code [33]. We include contributions from the messenger particles into the beta functions in the renormalization-group evolution from the messenger scale to the GUT scale. $M_{2-3}$ and $M_{1-2}$ are obtained and are shown in Fig. 5. It is clear from Fig. 5 that the ranges of $M_{2-3}$ and $M_{1-2}$ are almost the same in the MSUGRA and GMSB parameter spaces. Therefore, we conclude that there is little effect that comes purely from the difference between the SUSY-particle spectra in MSUGRA and GMSB.

The gaugino masses satisfy the GUT relation in both the MSUGRA and GMSB spectra, which may be the reason why $M_{2-3}$ and $M_{1-2}$ are almost the same in the two spectra. The gaugino mass spectrum, however, might not satisfy the GUT relation. ${ }^{27}$ Even in this case, we can obtain the upper bound of the lifetime through $M_{2-3}$ for the $\mathrm{SU}(5)_{\mathrm{GUT}} \times \mathrm{U}(2)_{H}$ model and through $M_{1-2}$ for the $\mathrm{SU}(5)_{\mathrm{GUT}} \times \mathrm{U}(3)_{H}$ model.

\section{Vectorlike $\mathrm{SU}(5)_{\text {GUT }}$ multiplet at low energy}

There are several motivations to consider charged particles in vectorlike representations, whose masses are of the order of the SUSY-breaking scale or an intermediate scale. Messenger particles are necessary in the GMSB models, and the anomaly cancellation of the discrete $R$ symmetry also requires [39] extra particles such as $\mathrm{SU}(5)_{\mathrm{GUT}}-\left(\mathbf{5}+\mathbf{5}^{*}\right)$.

There are three effects on the proton lifetime in the presence of these particles. The first two effects come from the changes in the values of the unified coupling constant $\alpha_{\text {GUT }}$ and the renormalization factor $A_{R}$ of the proton-decay operators. First, the unified gauge-coupling constant is larger in the presence of new particles, and hence the decay rate is enhanced. Then, the lifetime is shortened by a factor not smaller than 0.66 when a vectorlike pair $\mathrm{SU}(5)_{\mathrm{GUT}^{-}}(\mathbf{5}$ $+\mathbf{5}^{*}$ ) exists at an energy scale not lower than $1 \mathrm{TeV}$. Second, the renormalization factor $A_{R}$ is changed by such a vectorlike pair only in its short-distance part. The new expression for $A_{R}^{(\mathrm{SD})}$ is now given by

$$
\begin{aligned}
A_{R}^{(\mathrm{SD})}= & \left(\frac{\alpha_{C}\left(M_{Z}\right)}{\alpha_{C}(M)}\right)^{(4 / 3) / 3}\left(\frac{\alpha_{L}\left(M_{Z}\right)}{\alpha_{L}(M)}\right)^{(3 / 2) /(-1)} \\
& \times\left(\frac{\alpha_{Y}\left(M_{Z}\right)}{\alpha_{Y}(M)}\right)^{(23 / 30) /(-33 / 5)}\left(\frac{\alpha_{C}(M)}{\alpha_{C}\left(M_{G}\right)}\right)^{(4 / 3) / 2} \\
& \times\left(\frac{\alpha_{L}(M)}{\alpha_{L}\left(M_{G}\right)}\right)^{(3 / 2) /(-2)}\left(\frac{\alpha_{Y}(M)}{\alpha_{Y}\left(M_{G}\right)}\right)^{(23 / 30) /(-38 / 5)},
\end{aligned}
$$

where $M$ is the mass scale of the vectorlike pair. We find that $A_{R}^{(\mathrm{SD})}$ increases from 2.1 to 2.5 as the mass scale $M$ decreases from the GUT scale to $1 \mathrm{TeV}$. Thus, the lifetime is shortened by a factor not smaller than 0.71 because of the renormalization factor.

The third effect is due to threshold corrections from the vectorlike particles. The triplets and doublets in the vectorlike pair $\mathbf{5}^{+} \mathbf{5}^{*}$ are expected to have different masses, just as the bottom quark and tau lepton do. The triplets will be heavier than the doublets by

$$
\frac{M_{\mathbf{3}+3} *}{M_{\mathbf{2}+\mathbf{2} *}} \simeq\left(\frac{\alpha_{C}\left(M_{\mathbf{5}+\mathbf{5}^{*}}\right)}{\alpha_{C}\left(M_{G}\right)}\right)^{4 / 3},
$$

which increases from 1.0 to 2.1 as the mass scale $M_{5+5^{*}}$ of a vectorlike pair $\mathrm{SU}(5)_{\mathrm{GUT}^{-}}\left(\mathbf{5}+\mathbf{5}^{*}\right)$ decreases from the GUT scale to $1 \mathrm{TeV}$. The upper bound of the proton lifetime in the $\mathrm{SU}(5)_{\mathrm{GUT}} \times \mathrm{U}(2)_{H}$ model becomes tighter by a factor of $\left(M_{2+2} * M_{3+3}\right) \geq 0.48$ as $M_{2-3}$ is decreased by a factor of

\footnotetext{
${ }^{27}$ Gaugino masses without the GUT relation are not unnatural at all in the product-group unification models we discuss in this article [38].
} 
$\left(M_{2+2} / M_{3+3}\right)^{1 / 4}$; in the $\mathrm{SU}(5)_{\mathrm{GUT}} \times \mathrm{U}(3)_{H}$ model, instead, it is loosened by a factor $\left(M_{3+3} * / M_{2+2} *\right)^{2 / 7} \lesssim 1.2$ as $M_{1-2}$ is increased.

The proton decay is thus enhanced by all three effects in the $\mathrm{SU}(5)_{\mathrm{GUT}} \times \mathrm{U}(2)_{H}$ model; the lifetime is shortened by a factor of 0.22 when $\mathrm{SU}(5)_{\mathrm{GUT}^{-}}\left(\mathbf{5}+\mathbf{5}^{*}\right)$ exists at $1 \mathrm{TeV}$. The rate is also enhanced in the $\mathrm{SU}(5)_{\mathrm{GUT}} \times \mathrm{U}(3)_{H}$ model; the lifetime is shortened by a factor of 0.56 .

\section{CONCLUSIONS AND DISCUSSION}

We analyzed the proton-decay amplitude in a class of models of SUSY GUTs: $\mathrm{SU}(5)_{\mathrm{GUT}} \times \mathrm{U}(N)_{H}$ models with $N$ $=2,3$. Dimension-5 proton-decay operators are completely forbidden, and hence the gauge-boson exchange is the process that dominates the proton decay. We found that the gauge-boson mass is bounded from above by

$$
M_{G} \lesssim 0.40 \times 10^{-0.82 \times c} \times M_{2-3}
$$

in the $\mathrm{SU}(5)_{\mathrm{GUT}} \times \mathrm{U}(2)_{H} \operatorname{model}^{28}$ and by

$$
M_{G} \lesssim 0.60 \times 10^{-0.4 \times c} \times M_{1-2}
$$

in the $\mathrm{SU}(5)_{\mathrm{GUT}} \times \mathrm{U}(3)_{H}$ model. Here, $M_{2-3}\left[M_{1-2}\right]$ denotes an energy scale where $\mathrm{SU}(2)_{L}$ and $\mathrm{SU}(3)_{C}\left[\mathrm{U}(1)_{Y}\right.$ and $\mathrm{SU}(2)_{L}$ ] gauge-coupling constants are equal. In the right-hand sides, $c$ are coefficients of the nonrenormalizable operator (22) in the $\mathrm{SU}(5)_{\mathrm{GUT}} \times \mathrm{U}(2)_{H}$ model and of the one that corresponds to Eq. (22) in the $\mathrm{SU}(5)_{\mathrm{GUT}} \times \mathrm{U}(3)_{H}$ model. It is quite important to note that the upper bound was obtained in these models (for fixed $c$ ), which leads to the upper bound of the lifetime. Although the gauge-boson masses are bounded from below also in the latter model, the lower bound is of no importance. This is because it predicts a lifetime much shorter than the lower bound obtained so far from experiments.

The coefficients $c$ directly affect the gauge-coupling unification and hence they appear in the above formulas. One will see later that they are the largest source of uncertainties in the upper bound of the lifetime if $c$ are of the order of unity. Although there may be an extra (broken) symmetry or any dynamics that suppress the nonrenormalizable operators, we leave $c$ in the formulas for generic cases.

In Sec. I, we briefly mentioned two other classes of models of SUSY GUTs constructed in four-dimensional spacetime. Let us make a brief summary of the mass of the gauge bosons of such models before we proceed to a discussion of the lifetime.

Let us first discuss the gauge-boson mass in the models in $[10,11]$. The spectrum around the GUT scale consists of three $\left((\mathbf{a d j}, \mathbf{1})^{0}+(\mathbf{1}, \mathbf{a d j})^{0}\right)$ 's and two $\left((\mathbf{3}, \mathbf{2})^{-5 / 6}+\right.$ H.c. $)$ 's of the MSSM gauge group, in addition to the GUT gauge boson. The parameters of the models allow a spectrum where the matter particles are lighter than the GUT gauge boson. Then, the one-loop threshold corrections from such a spec-

\footnotetext{
${ }^{28}$ This expression for the upper bound of $M_{G}$ is valid as long as $c \gtrsim-0.16+1.3 \times\left(\epsilon_{g} / \alpha_{\mathrm{GUT}}\right)$.
}

trum imply that the GUT gauge-boson mass is heavier than the energy scale of approximate unification [42]. Therefore, no upper bound is virtually obtained in the models in $[10,11]$. A lower bound might be obtained, yet no full study has been done so far. Nonrenormalizable operators in the gauge kinetic functions affect the matching equations just as in our analysis.

On the contrary, in the models in [18], nonrenormalizable operators do not affect the matching equations and, moreover, the mass of the GUT gauge boson is smaller than the energy scale where the three gauge-coupling constants are approximately unified:

$$
M_{G} \sim \lambda^{a} M_{\text {unif }},
$$

where $M_{\text {unif }} \sim M_{2-3} \sim M_{1-2}, \lambda$ is a small parameter of the order of $10^{-1}$, and $a$ is the charge of a field whose VEV breaks the $\mathrm{SU}(5)_{\mathrm{GUT}}$ symmetry down to $\mathrm{SU}(3)_{C} \times \mathrm{SU}(2)_{L}$ $\times \mathrm{U}(1)_{Y}$. Thus, $M_{G}$ is fairly small in the models. The upper bound would be obtained once a model ( $\lambda$ and $a$, in particular) is fixed. The Super-Kamiokande experiment already puts constraints on the choices of $\lambda$ and $a$. The proton decays also through dimension-5 operators, although these operators can be suppressed in some models in this class.

Thus, the ranges of the proton lifetime of those models lie in the following order:

$$
\begin{aligned}
\tau([18])_{\mathrm{dim} 6} & \sim \tau\left(\mathrm{SU}(5)_{\mathrm{GUT}} \times \mathrm{U}(2)_{H}\right) \\
& \lesssim \tau\left(\mathrm{SU}(5)_{\mathrm{GUT}} \times \mathrm{U}(3)_{H}\right) \lesssim \tau([10],[11]) .
\end{aligned}
$$

However, the ranges would have a certain amount of overlap between one another, and hence it would be impossible to single out a model only from the decay rate of the proton. Detailed information on the branching ratio of various decay modes does not help for that purpose either; the decay is induced in all the above models ${ }^{29}$ by one and the same ${ }^{30}$ mechanism: the gauge-boson exchange.

Even if no model can be singled out, one can and will be able to exclude some of the models on the basis of experimental results currently available and obtained in the future, respectively. We summarize, in the following, the upper bound of the proton lifetime for the $\mathrm{SU}(5)_{\mathrm{GUT}} \times \mathrm{U}(2)_{H}$ model and the $\mathrm{SU}(5)_{\mathrm{GUT}} \times \mathrm{U}(3)_{H}$ model. It would also be of importance if one finds an upper bound and a lower bound of the lifetime in the models in [18] and in [10-12], respectively.

Now the proton lifetime is bounded from above by

\footnotetext{
${ }^{29}$ The proton decay can be induced by the gauge-boson exchange also in SUSY GUT models in higher-dimensional spacetime [5,7]. The branching ratio of various modes can be different $[14,15]$ from the standard one in those models.

${ }^{30}$ If the dimension- 5 decay is not the dominant process in the last class of models.
} 


$$
\begin{aligned}
\tau\left(p \rightarrow \pi^{0} e^{+}\right) \lesssim & 4.1 \times 10^{32}\left(\frac{M_{2-3}}{10^{15.8} \mathrm{GeV}}\right)^{4}\left(10^{-3.2 c}\right) \\
& \times\left(\frac{0.015 \mathrm{GeV}^{3}}{\alpha_{H}}\right)^{2}\left(\frac{1}{25 \alpha_{\mathrm{GUT}}}\right)^{2}\left(\frac{2.5}{A_{R}}\right)^{2} \mathrm{yr}
\end{aligned}
$$

in the $\mathrm{SU}(5)_{\mathrm{GUT}} \times \mathrm{U}(2)_{H}$ model. The largest uncertainty in this prediction comes from the value of $c$ and another from the systematic error in $\alpha_{H}$. No estimate is available for the value of $c$. The error in $\alpha_{H}$ is not studied very much, yet the lifetime is changed by a factor of $(0.5-2)$ if the conservative estimate in [35] is adopted. ${ }^{31}$ The experimental value of the QCD coupling constant $\alpha_{s}^{\overline{\mathrm{MS}},(5)}\left(M_{Z}\right)$ changes the prediction through the change in $M_{2-3}$. The upper bound is changed by a factor of (0.15-5.9) when the coupling constant is varied by $\pm 2 \sigma$ error determined by experiments. The threshold corrections from SUSY particles also changes the prediction through the change in $M_{2-3}$. They change $M_{2-3}$ typically from $10^{15.77} \mathrm{GeV}$ to $10^{15.90} \mathrm{GeV}$, and hence the upper bound is changed by a factor of $(0.75-2.5)$. Therefore, the theoretical upper bound exceeds the experimental lower bound $\left[\tau\left(p \rightarrow \pi^{0} e^{+}\right) \geq 5 \times 10^{33} \mathrm{yr} ; 90 \%\right.$ C.L. $]$ only when ${ }^{32}$ all the low-energy uncertainties are exploited. See Sec. III C for the uncertainties that arise in the way of our analysis.

The lifetime is shortened by a factor not smaller than 0.22 if $\mathrm{SU}(5)_{\mathrm{GUT}^{-}}\left(\mathbf{5}+\mathbf{5}^{*}\right)$ exists at low energy. The threshold corrections from these particles contribute by a factor not smaller than 0.47 through the change in $M_{2-3}$, and the changes in $\alpha_{\mathrm{GUT}}$ and in $A_{R}$ contribute by factors not smaller than 0.66 and 0.71 , respectively. Thus, those particles at 1
$\mathrm{TeV}$ would hardly be reconciled with the experimental bound without incorporating the nonrenormalizable operator (22).

The lifetime is bounded from above by

$$
\begin{aligned}
\tau\left(p \rightarrow \pi^{0} e^{+}\right) \lesssim & 2.1 \times 10^{35}\left(\frac{M_{1-2}}{10^{16.3} \mathrm{GeV}}\right)^{4}\left(10^{-2 c}\right) \\
& \times\left(\frac{0.015 \mathrm{GeV}^{3}}{\alpha_{H}}\right)^{2}\left(\frac{1}{25 \alpha_{\mathrm{GUT}}}\right)^{2}\left(\frac{2.5}{A_{R}}\right)^{2} \mathrm{yr}
\end{aligned}
$$

in the $\mathrm{SU}(5)_{\mathrm{GUT}} \times \mathrm{U}(3)_{H}$ model. Uncertainties arise $\mathrm{e}^{33}$ from $c$ and $\alpha_{H}$ as in the previous model. The value of the QCD coupling constant is not relevant to the prediction. The SUSY threshold corrections change the upper bound typically by a factor of $(0.40-2.5)$ as $M_{1-2}$ changes from $10^{16.20} \mathrm{GeV}$ to $10^{16.40} \mathrm{GeV}$.

The lifetime is shortened by a factor not smaller than 0.56 in the presence of $\mathrm{SU}(5)_{\mathrm{GUT}^{-}}\left(\mathbf{5}+\mathbf{5}^{*}\right)$ below the GUT scale. The decay is more enhanced as their mass is smaller. The enhancement factor 0.56 (when the mass is $1 \mathrm{TeV}$ ) consists of a suppression factor 1.2 , which comes from $M_{1-2}$ changed by the threshold corrections of these particles, and enhancement factors 0.66 and 0.71 , respectively, from $\alpha_{\mathrm{GUT}}$ and $A_{R}$.

\section{ACKNOWLEDGMENTS}

The authors are grateful to the Theory Division of CERN, where the early part of this work was done, for hospitality. They thank T. Yanagida for discussions and a careful reading of this manuscript. T.W. thanks the Japan Society for the Promotion of Science for financial support.

\section{APPENDIX A: RENORMALIZATION-GROUP EQUATIONS}

In this appendix, the renormalization-group equations of the coupling constants of the models are listed.

\section{1. $\mathrm{SU}(5)_{\mathrm{GUT}} \times \mathrm{U}(2)_{H}$ model}

$$
\begin{aligned}
\frac{\partial}{\partial \ln \mu}\left(\frac{1}{\alpha_{2 H}}(\mu)\right)= & \frac{-2}{2 \pi} \text { (one loop) }-\frac{2}{2 \pi} \frac{\left(6 \alpha_{2 H}-5 \alpha_{2 H}^{\lambda}-\alpha_{2 H}^{\lambda^{\prime}}\right)}{2 \pi}-\frac{1}{2 \pi} \frac{6\left(3 \alpha_{2 H}+\alpha_{1 H}\right)-5\left(3 \alpha_{2 H}^{\lambda}+\alpha_{1 H}^{\lambda}\right)-\left(3 \alpha_{2 H}^{\lambda^{\prime}}+\alpha_{1 H}^{\lambda^{\prime}}\right)}{4 \pi} \\
& -\frac{5}{2 \pi}\left(\frac{24 / 10}{\pi} \alpha_{\mathrm{GUT}}\right) \cdot \\
\frac{\partial}{\partial \ln \mu}\left(\frac{1}{\alpha_{2 H}^{\lambda}}(\mu)\right)= & \frac{-2}{2 \pi}\left(\frac{\alpha_{2 H}}{\alpha_{2 H}^{\lambda}}\right)+\frac{1}{\alpha_{2 H}^{\lambda}} \frac{\left(6 \alpha_{2 H}-5 \alpha_{2 H}^{\lambda}-\alpha_{2 H}^{\lambda^{\prime}}\right)}{2 \pi}+\frac{2}{\alpha_{2 H}^{\lambda}} \frac{\left(3 \alpha_{2 H}+\alpha_{1 H}\right)-\left(3 \alpha_{2 H}^{\lambda}+\alpha_{1 H}^{\lambda}\right)}{4 \pi}+\frac{2}{\alpha_{2 H}^{\lambda}}\left(\frac{24 / 10}{\pi} \alpha_{\mathrm{GUT}}\right) .
\end{aligned}
$$

\footnotetext{
${ }^{31}$ We presented a numerical value of the upper bound of the proton lifetime in the Abstract of this article. These two uncertainties are not included there, since it is impossible to make a precise estimate of them at this moment. The following two uncertainties, on the other hand, are included in obtaining the numerical value in the Abstract.

${ }^{32}$ This statement holds as long as the nonrenormalizable operator (22) is neglected, i.e., as long as $c \gg-1$.

${ }^{33}$ They are not included in obtaining the numerical value in the Abstract just for the same reason as in the previous model.
} 


$$
\begin{aligned}
& \frac{\partial}{\partial \ln \mu}\left(\frac{1}{\alpha_{2 H}^{\lambda^{\prime}}}(\mu)\right)=\frac{-2}{2 \pi}\left(\frac{\alpha_{2 H}}{\alpha_{2 H}^{\lambda^{\prime}}}\right)+\frac{1}{\alpha_{2 H}^{\lambda^{\prime}}} \frac{\left(6 \alpha_{2 H}-5 \alpha_{2 H}^{\lambda}-\alpha_{2 H}^{\lambda^{\prime}}\right)}{2 \pi}+\frac{2}{\alpha_{2 H}^{\lambda^{\prime}}} \frac{\left(3 \alpha_{2 H}+\alpha_{1 H}\right)-\left(3 \alpha_{2 H}^{\lambda^{\prime}}+\alpha_{1 H}^{\lambda^{\prime}}\right)}{4 \pi} . \\
& \frac{\partial}{\partial \ln \mu}\left(\frac{1}{\alpha_{1 H}}(\mu)\right)=\frac{-6}{2 \pi} \text { (one loop) }-\frac{1}{2 \pi} \frac{6\left(3 \alpha_{2 H}+\alpha_{1 H}\right)-5\left(3 \alpha_{2 H}^{\lambda}+\alpha_{1 H}^{\lambda}\right)-\left(3 \alpha_{2 H}^{\lambda^{\prime}}+\alpha_{1 H}^{\lambda^{\prime}}\right)}{4 \pi}-\frac{5}{2 \pi}\left(\frac{24 / 10}{\pi} \alpha_{\mathrm{GUT}}\right) \\
& \frac{\partial}{\partial \ln \mu}\left(\frac{1}{\alpha_{1 H}^{\lambda}}(\mu)\right)=\frac{-6}{2 \pi}\left(\frac{\alpha_{1 H}}{\alpha_{1 H}^{\lambda}}\right)+\frac{1}{\alpha_{1 H}^{\lambda}} \frac{\left(6 \alpha_{1 H}-5 \alpha_{1 H}^{\lambda}-\alpha_{1 H}^{\lambda^{\prime}}\right)}{2 \pi}+\frac{2}{\alpha_{1 H}^{\lambda}} \frac{\left(3 \alpha_{2 H}+\alpha_{1 H}\right)-\left(3 \alpha_{2 H}^{\lambda}+\alpha_{1 H}^{\lambda}\right)}{4 \pi}+\frac{2}{\alpha_{1 H}^{\lambda}}\left(\frac{24 / 10}{\pi} \alpha_{\mathrm{GUT}}\right) . \\
& \frac{\partial}{\partial \ln \mu}\left(\frac{1}{\alpha_{1 H}^{\lambda^{\prime}}}(\mu)\right)=\frac{-6}{2 \pi}\left(\frac{\alpha_{1 H}}{\alpha_{1 H}^{\lambda^{\prime}}}\right)+\frac{1}{\alpha_{1 H}^{\lambda^{\prime}}} \frac{\left(6 \alpha_{1 H}-5 \alpha_{1 H}^{\lambda}-\alpha_{1 H}^{\lambda^{\prime}}\right)}{2 \pi}+\frac{2}{\alpha_{1 H}^{\lambda^{\prime}}} \frac{\left(3 \alpha_{2 H}+\alpha_{1 H}\right)-\left(3 \alpha_{2 H}^{\lambda^{\prime}}+\alpha_{1 H}^{\lambda^{\prime}}\right)}{4 \pi} .
\end{aligned}
$$

\section{2. $\mathrm{SU}(5)_{\mathrm{GUT}} \times \mathrm{U}(3)_{H}$ model}

$$
\begin{aligned}
\frac{\partial}{\partial \ln \mu}\left(\frac{1}{\alpha_{3 H}}(\mu)\right)= & 0 \text { (one loop) }-\frac{3}{2 \pi} \frac{\left(6 \alpha_{3 H}-5 \alpha_{3 H}^{\lambda}-\alpha_{3 H}^{\lambda^{\prime}}\right)}{2 \pi}-\frac{1}{2 \pi} \frac{6\left(8 \alpha_{3 H}+\alpha_{1 H}\right)-5\left(8 \alpha_{3 H}^{\lambda}+\alpha_{1 H}^{\lambda}\right)-\left(8 \alpha_{3 H}^{\lambda^{\prime}}+\alpha_{1 H}^{\lambda^{\prime}}\right)}{6 \pi} \\
& +\left(-\frac{5}{2 \pi} \frac{24 / 10}{\pi} \alpha_{\mathrm{GUT}}+\frac{10}{2 \pi} \frac{1}{2 \pi} \alpha_{h}\right) \\
\frac{\partial}{\partial \ln \mu}\left(\frac{1}{\alpha_{3 H}^{\lambda}}(\mu)\right)= & 0+\frac{1}{\alpha_{3 H}^{\lambda}} \frac{\left(6 \alpha_{3 H}-5 \alpha_{3 H}^{\lambda}-\alpha_{3 H}^{\lambda^{\prime}}\right)}{2 \pi}+\frac{2}{\alpha_{3 H}^{\lambda}} \frac{\left(8 \alpha_{3 H}+\alpha_{1 H}\right)-\left(8 \alpha_{3 H}^{\lambda}+\alpha_{1 H}^{\lambda}\right)}{6 \pi}+\frac{2}{\alpha_{3 H}^{\lambda}}\left(\frac{24 / 10}{\pi} \alpha_{\mathrm{GUT}}-\frac{1}{2 \pi} \alpha_{h}\right) \\
\frac{\partial}{\partial \ln \mu}\left(\frac{1}{\alpha_{3 H}^{\lambda^{\prime}}}(\mu)\right)= & 0+\frac{1}{\alpha_{3 H}^{\lambda^{\prime}}} \frac{\left(6 \alpha_{3 H}-5 \alpha_{3 H}^{\lambda}-\alpha_{3 H}^{\lambda^{\prime}}\right)}{2 \pi}+\frac{2}{\alpha_{3 H}^{\lambda^{\prime}}} \frac{\left(8 \alpha_{3 H}+\alpha_{1 H}\right)-\left(8 \alpha_{3 H}^{\lambda^{\prime}}+\alpha_{1 H}^{\lambda^{\prime}}\right)}{6 \pi}+\frac{2}{\alpha_{3 H}^{\lambda^{\prime}}}\left(-\frac{5}{2 \pi} \alpha_{h}\right)
\end{aligned}
$$$$
\frac{\partial}{\partial \ln \mu}\left(\frac{1}{\alpha_{1 H}}(\mu)\right)=\frac{-6}{2 \pi} \text { (one loop) }-\frac{1}{2 \pi} \frac{6\left(8 \alpha_{3 H}+\alpha_{1 H}\right)-5\left(8 \alpha_{3 H}^{\lambda}+\alpha_{1 H}^{\lambda}\right)-\left(8 \alpha_{3 H^{\prime}}^{\lambda^{\prime}}+\alpha_{1 H}^{\lambda^{\prime}}\right)}{6 \pi}
$$$$
+\left(-\frac{5}{2 \pi} \frac{24 / 10}{\pi} \alpha_{\mathrm{GUT}}+\frac{10}{2 \pi} \frac{1}{2 \pi} \alpha_{h}\right) .
$$$$
\frac{\partial}{\partial \ln \mu}\left(\frac{1}{\alpha_{1 H}^{\lambda}}(\mu)\right)=\frac{-6}{2 \pi}\left(\frac{\alpha_{1 H}}{\alpha_{1 H}^{\lambda}}\right)+\frac{1}{\alpha_{1 H}^{\lambda}} \frac{\left(6 \alpha_{1 H}-5 \alpha_{1 H}^{\lambda}-\alpha_{1 H}^{\lambda^{\prime}}\right)}{2 \pi}+\frac{2}{\alpha_{1 H}^{\lambda}} \frac{\left(8 \alpha_{3 H}+\alpha_{1 H}\right)-\left(8 \alpha_{3 H}^{\lambda}+\alpha_{1 H}^{\lambda}\right)}{6 \pi}
$$$$
+\frac{2}{\alpha_{1 H}^{\lambda}}\left(\frac{24 / 10}{\pi} \alpha_{\mathrm{GUT}}-\frac{1}{2 \pi} \alpha_{h}\right) \text {. }
$$$$
\frac{\partial}{\partial \ln \mu}\left(\frac{1}{\alpha_{1 H}^{\lambda^{\prime}}}(\mu)\right)=\frac{-6}{2 \pi}\left(\frac{\alpha_{1 H}}{\alpha_{1 H}^{\lambda^{\prime}}}\right)+\frac{1}{\alpha_{1 H}^{\lambda^{\prime}}} \frac{\left(6 \alpha_{1 H}-5 \alpha_{1 H}^{\lambda}-\alpha_{1 H}^{\lambda^{\prime}}\right)}{2 \pi}+\frac{2}{\alpha_{1 H}^{\lambda^{\prime}}} \frac{\left(8 \alpha_{3 H}+\alpha_{1 H}\right)-\left(8 \alpha_{3 H}^{\lambda^{\prime}}+\alpha_{1 H}^{\lambda^{\prime}}\right)}{6 \pi}+\frac{2}{\alpha_{1 H}^{\lambda^{\prime}}}\left(-\frac{5}{2 \pi} \alpha_{h}\right) .
$$$$
\frac{\partial}{\partial \ln \mu}\left(\frac{1}{\alpha_{h}}(\mu)\right)=\frac{1}{\alpha_{h}} \frac{2\left(8 \alpha_{3 H}+\alpha_{1 H}\right)-\left(8 \alpha_{3 H}^{\lambda}+\alpha_{1 H}^{\lambda}\right)-\left(8 \alpha_{3 H}^{\lambda^{\prime}}+\alpha_{1 H}^{\lambda^{\prime}}\right)}{6 \pi}+\left(\frac{1}{\alpha_{h}}\right)\left(\frac{2 \times 24 / 10}{\pi} \alpha_{\mathrm{GUT}}-\frac{(1+5+3)}{2 \pi} \alpha_{h}\right) .
$$ 


\section{APPENDIX B: $\mathcal{N}=2$ SUSY AND INFRARED-FIXED RENORMALIZATION-GROUP FLOW}

The particle content in the GUT-symmetry-breaking sector of the $\mathrm{SU}(5)_{\mathrm{GUT}} \times \mathrm{U}(2)_{H}$ model can be regarded as multiplets of the $\mathcal{N}=2$ SUSY [17], and $\mathcal{N}=2$ SUSY is enhanced in this sector [8] when the $\mathrm{SU}(5)_{\mathrm{GUT}}$ gauge interaction is neglected and the coupling constants satisfy

$$
g_{1 H} \simeq \lambda_{1 H}\left(\sim \lambda_{1 H}^{\prime}\right), \quad g_{2 H} \simeq \lambda_{2 H}\left(\sim \lambda_{2 H}^{\prime}\right)
$$

One can see in the right panel of Fig. 2 that the parameter region survives in the presence of the two-loop effects only when the $\mathcal{N}=2$ SUSY is approximately preserved; the $M_{3 V}$ $\simeq M_{3 C}$ line is equivalent to $\alpha_{2 H} \simeq \alpha_{2 H}^{\lambda^{\left({ }^{\prime}\right)}}$ when the $\mathrm{SU}(5)_{\mathrm{GUT}}$ gauge interaction is neglected.

This is not a coincidence. In any gauge theory with $\mathcal{N}$ $=2$ SUSY, gauge-coupling constants are renormalized only at the one-loop level [26]. Anomalous dimensions of hypermultiplets vanish [28] at all orders in a perturbative expansion, and even nonperturbatively. Therefore, the parameter allowed in the one-loop analysis is still allowed when $\mathcal{N}$ $=2$ SUSY is preserved even after the two-loop effects have also been taken into account.

The band of the region around the $\mathcal{N}=2$ SUSY line almost becomes a line as $M_{G}$ becomes larger. For parameters above that line, the $\alpha_{2 H}$ coupling constant becomes large at a renormalization point lower than $M_{3 V}$, while for parameters below the line, the $\alpha_{2 H}^{\lambda}$ coupling becomes large at a renormalization point lower than $M_{3 C}$; a viable set of parameters was not found for large $M_{G}$ even on the line in our numerical calculation. It does not mean, however, that the parameter does not exist at all on the $\mathcal{N}=2$ SUSY line $\left(M_{3 V} \simeq M_{3 C}\right)$ for large $M_{G}$, as seen below. The $\mathcal{N}=2$ SUSY relations (B1) are not only renormalization-group invariant but also infrared- (IR-) fixed relations of the renormalization group:

$$
\begin{aligned}
\frac{\partial}{\partial \ln \mu}\left(\frac{1}{\alpha_{2 H}}-\frac{1}{\alpha_{2 H}^{\lambda}}\right)(\mu)= & \left(\frac{21}{2 \pi}+\frac{7}{\alpha_{2 H}^{\lambda}}\right) \frac{\left(\alpha_{2 H}^{\lambda}-\alpha_{2 H}\right)}{2 \pi} \\
& +\left(\frac{6}{2 \pi}+\frac{2}{\alpha_{2 H}^{\lambda}}\right) \frac{\left(\alpha_{1 H}^{\lambda}-\alpha_{1 H}\right)}{4 \pi},
\end{aligned}
$$

$$
\begin{aligned}
& \frac{\partial}{\partial \ln \mu}\left(\frac{1}{\alpha_{1 H}}-\frac{1}{\alpha_{1 H}^{\lambda}}\right)(\mu) \\
& =\left(\frac{6}{2 \pi}+\frac{2}{\alpha_{1 H}^{\lambda}}\right) \frac{3\left(\alpha_{2 H}^{\lambda}-\alpha_{2 H}\right)+\left(\alpha_{1 H}^{\lambda}-\alpha_{1 H}\right)}{4 \pi},
\end{aligned}
$$

where the SU(5) $)_{\text {GUT }}$ interaction is still neglected. This implies that the renormalization-group evolution to the UV im- mediately becomes unstable,$^{34}$ even for a set of parameters that is slightly distant from the IR-fixed relations. The IRfixed property (UV instability) also implies that the parameter region is thin only when $M=M_{G}$, and not when the coupling constants are evaluated at $M \gg M_{G}$.

Thus, we can expect that the one-loop analysis is completely reliable for a set of parameters exactly on the $\mathcal{N}=2$ SUSY line and, in particular, that a viable set of parameters does exist on the line even if it is not found in the numerical analysis. Therefore, the maximum value of $M_{G}$ is given at the point indicated by an arrow in the right panel of Fig. 2 . At least, there would be no doubt that the maximum value of $M_{G}$ obtained in such a way provides a conservative upper bound of $M_{G}$.

The above argument, however, is correct only when the $\mathrm{SU}(5)_{\text {GUT }}$ gauge interaction is neglected. Therefore, let us now discuss the effects of the $\mathrm{SU}(5)_{\text {GUT }}$ gauge interaction. These break the $\mathcal{N}=2$ SUSY in the sector. Thus, the $\mathcal{N}=2$ SUSY relations in Eq. (B1) are no longer renormalizationgroup invariant, and the renormalization-group flow is no longer one-loop exact. However, the $\mathrm{SU}(5)_{\mathrm{GUT}}$ interaction is much weaker than the $\mathrm{U}(2)_{H}$ interactions and its effects are small. ${ }^{35}$ Thus, they can be treated as small perturbations to the $\mathcal{N}=2$ SUSY flow. In particular, the IR-fixed property of the renormalization-group equations (A1)-(A6) is not changed, ${ }^{36}$ except that the IR-fixed relations are slightly modified to

$$
\begin{gathered}
\left(\alpha_{2 \mathrm{H}}-\alpha_{2 \mathrm{H}}^{\lambda}\right), \quad\left(\alpha_{2 \mathrm{H}}-\alpha_{2 \mathrm{H}}^{\lambda^{\prime}}\right), \quad\left(\alpha_{1 \mathrm{H}}-\alpha_{1 \mathrm{H}}^{\lambda}\right), \\
\left(\alpha_{1 \mathrm{H}}-\alpha_{1 \mathrm{H}}^{\lambda^{\prime}}\right) \simeq \mathcal{O}\left(\alpha_{\mathrm{GUT}}\right) .
\end{gathered}
$$

The coupling constants flow down to the modified fixed relations and then follow the relations. Thus, the evolution of the coupling constants toward the UV is most stable when the parameter satisfies the "IR-fixed relations." The modified fixed relations are still almost the $\mathcal{N}=2$ SUSY relations, and

\footnotetext{
${ }^{34}$ This is the reason we believe that it would not make the parameter region wider even if we set the values of the undetermined parameters $\alpha_{2 H}^{\lambda^{\prime}}$ and $\alpha_{1 H}^{\lambda^{\left({ }^{\prime}\right)}}$ differently from those in Eq. (16). A deviation from the $\mathcal{N}=2$ SUSY relation at $M=M_{G}$ would immediately lead to an UV-unstable behavior in the renormalization-group evolution.

${ }^{35}$ This can be seen from the fact that the parameter region is still almost around the $\mathcal{N}=2$ SUSY line, i.e., $M_{3 V} \simeq M_{3 C}$, in the right panel of Fig. 2. $(\mathcal{N}=2)$-SUSY-breaking interactions are included in the figure.

${ }^{36}$ There is no IR-fixed relation in its strict meaning in the presence of the SU(5) $)_{\text {GUT }}$ interaction. The "IR-fixed relations" in Eq. (B4) involve $\alpha_{2 H}^{\lambda^{\left({ }^{\prime}\right)}}$ and $\alpha_{1 H}^{\lambda^{\left({ }^{\prime}\right)}}$ on the right-hand sides, and hence the "fixed relations" themselves change as the coupling constants flow. However, we still consider that they are almost IR-fixed relations, because the beta functions of the quantities in the right-hand sides $\left[\simeq \mathcal{O}\left(\alpha_{\mathrm{GUT}}\right)\right]$ are much smaller than those of the quantities in the left-hand sides.
} 
hence the one-loop evolution is almost correct for the parameter satisfying the relations; the beta functions are different from those at one loop only by an order of $\alpha_{\mathrm{GUT}}$. Moreover, combinations such as $\left(\alpha_{2 H}-\alpha_{2 H}^{\lambda^{\left({ }^{\prime}\right)}}\right)$ partially absorb the
$\mathrm{SU}(5)_{\mathrm{GUT}}$ contributions in the beta functions. Therefore, the corrections to the one-loop evolution are estimated conservatively from above when the $\mathrm{SU}(5)_{\mathrm{GUT}}$ contribution is purely added to the one-loop beta functions, as we did in Sec. III C.
[1] E. Witten, Nucl. Phys. B258, 75 (1985).

[2] N. Sakai and T. Yanagida, Nucl. Phys. B197, 533 (1982); S. Weinberg, Phys. Rev. D 26, 287 (1982).

[3] H. Murayama and A. Pierce, Phys. Rev. D 65, 055009 (2002), and references therein.

[4] Super-Kamiokande Collaboration, Y. Hayato et al., Phys. Rev. Lett. 83, 1529 (1999).

[5] Y. Kawamura, Prog. Theor. Phys. 105, 999 (2001).

[6] L.J. Hall and Y. Nomura, Phys. Rev. D 64, 055003 (2001).

[7] E. Witten, hep-ph/0201018.

[8] Y. Imamura, T. Watari, and T. Yanagida, Phys. Rev. D 64, 065023 (2001).

[9] T. Watari and T. Yanagida, Phys. Lett. B 520, 322 (2001); hep-ph/0208107.

[10] R. Barbieri, G.R. Dvali, and A. Strumia, Phys. Lett. B 333, 79 (1994).

[11] S.M. Barr, Phys. Rev. D 55, 6775 (1997).

[12] M. Dine, Y. Nir, and Y. Shadmi, Phys. Rev. D 66, 115001 (2002).

[13] I. Izawa and T. Yanagida, Prog. Theor. Phys. 97, 913 (1997).

[14] L.J. Hall and Y. Nomura, Phys. Rev. D 66, 075004 (2002).

[15] T. Friedmann and E. Witten, hep-th/0211269.

[16] T. Yanagida, Phys. Lett. B 344, 211 (1995).

[17] J. Hisano and T. Yanagida, Mod. Phys. Lett. A A 10, 3097 (1995).

[18] N. Maekawa, Prog. Theor. Phys. 106, 401 (2001); Phys. Lett. B 521, 42 (2001).

[19] K.I. Izawa, K. Kurosawa, Y. Nomura, and T. Yanagida, Phys. Rev. D 60, 115016 (1999), Appendix.

[20] N. Maekawa and T. Yamashita, Prog. Theor. Phys. 108, 719 (2002).

[21] M. Fujii and T. Watari, Phys. Lett. B 527, 106 (2002).

[22] T. Watari, Ph.D thesis, University of Tokyo, Tokyo, Japan, 2003.

[23] J. Hisano, H. Murayama, and T. Yanagida, Nucl. Phys. B402, 46 (1993)

[24] G.F. Giudice and A. Masiero, Phys. Lett. B 206, 480 (1988).
[25] I. Antoniadis, C. Kounnas, and K. Tamvakis, Phys. Lett. 119B, 377 (1982).

[26] M.T. Grisaru and W. Siegel, Nucl. Phys. B201, 292 (1982); B206, 496(E) (1982); P.S. Howe, K.S. Stelle, and P.C. West, Phys. Lett. 124B, 55 (1983).

[27] N. Arkani-Hamed and H. Murayama, J. High Energy Phys. 06, 030 (2000).

[28] R. Barbieri, S. Ferrara, L. Maiani, F. Palumbo, and C.A. Savoy, Phys. Lett. 115B, 212 (1982); B. de Wit, P.G. Lauwers, and A. Van Proeyen, Nucl. Phys. B255, 569 (1985); P.C. Argyres, M. Ronen Plesser, and N. Seiberg, ibid. B471, 159 (1996).

[29] Particle Data Group, K. Hagiwara et al., Phys. Rev. D 66, 010001 (2002).

[30] JLQCD Collaboration, S. Aoki et al., Phys. Rev. D 62, 014506 (2000).

[31] J. Hisano, hep-ph/0004266.

[32] L.E. Ibañez and C. Muñoz, Nucl. Phys. B245, 425 (1984).

[33] B.C. Allanach, Comput. Phys. Commun. 143, 305 (2002).

[34] B.C. Allanach, S. Kraml, and W. Porod, J. High Energy Phys. 03, 016 (2003).

[35] S. Raby, hep-ph/0211024.

[36] TITAND Working Group Collaboration, Y. Suzuki et al., hep-ex/0110005.

[37] Super-Kamiokande Collaboration, M. Shiozawa et al., Phys. Rev. Lett. 81, 3319 (1998).

[38] N. Arkani-Hamed, H.C. Cheng, and T. Moroi, Phys. Lett. B 387, 529 (1996).

[39] K. Kurosawa, N. Maru, and T. Yanagida, Phys. Lett. B 512, 203 (2001).

[40] LEP Higgs Working Group Collaboration, hep-ex/0107030.

[41] LEP II Supersymmetry Working Group, http:// lepsusy.web.cern.ch/lepsusy/www/inos_moriond01/charginos_ pub.html

[42] C. Bachas, C. Fabre, and T. Yanagida, Phys. Lett. B 370, 49 (1996). 